\title{
Outbreak COVID-19 in Medical Image Processing Using Deep Learning: A State-of-the-Art Review
}

\author{
Jaspreet $\operatorname{Kaur}^{1}$ (D) Prabhpreet Kaur $^{1}$
}

Received: 11 March 2021 / Accepted: 1 October 2021 / Published online: 19 October 2021

(c) CIMNE, Barcelona, Spain 2021

\begin{abstract}
From the month of December-19, the outbreak of Coronavirus (COVID-19) triggered several deaths and overstated every aspect of individual health. COVID-19 has been designated as a pandemic by World Health Organization. The circumstances placed serious trouble on every country worldwide, particularly with health arrangements and time-consuming responses. The increase in the positive cases of COVID-19 globally spread every day. The quantity of accessible diagnosing kits is restricted because of complications in detecting the existence of the illness. Fast and correct diagnosis of COVID-19 is a timely requirement for the prevention and controlling of the pandemic through suitable isolation and medicinal treatment. The significance of the present work is to discuss the outline of the deep learning techniques with medical imaging such as outburst prediction, virus transmitted indications, detection and treatment aspects, vaccine availability with remedy research. Abundant image resources of medical imaging as X-rays, Computed Tomography Scans, Magnetic Resonance imaging, formulate deep learning high-quality methods to fight against the pandemic COVID-19. The review presents a comprehensive idea of deep learning and its related applications in healthcare received over the past decade. At the last, some issues and confrontations to control the health crisis and outbreaks have been introduced. The progress in technology has contributed to developing individual's lives. The problems faced by the radiologists during medical imaging techniques and deep learning approaches for diagnosing the COVID-19 infections have been also discussed.
\end{abstract}

\section{Introduction}

The outbreak of COVID-19 and its associated inhibition complications have triggered a global health disaster affecting every segment of individual life. In the preliminary stages of the illness, the numerous people infected were very small, and it did not reflect the threat of this powerful capability so that most cases would resolve impulsively. With a steady sequence of instances, the WHO has announced that COVID-19 has become a pandemic with a tremendously high menace perspective that may affect the lives of millions of people in every country, especially with a pathetic health organization. The disease is poisonous because of two reasons: first, a novel virus included no vaccine has been found;

Jaspreet Kaur

jaspreet.kaur144@gmail.com

Prabhpreet Kaur

prabhpreet.cst@gndu.ac.in

1 Department of Computer Engineering \& Technology, Guru Nanak Dev University, Amritsar, Punjab, India second, effortlessly spread throughout directly or indirectly proximity with sick people.

The COVID-19 [1] worldometer statistics show reasons for great concern, through approximately $83,915,772$ people affected worldwide, and 1,827,949 patients lost in the fight and died on October 29, 2020. In addition to the alarming information, the USA is a solitary country that leads the standard carriers within health development, recording the uppermost quantity of COVID-19 sufferers pursued by India, Russia, South Africa, Brazil, and the record will go scheduled till 215 countries worldwide. In the USA, the total coronavirus cases are $102,169,665$, along with $74,014,468$ recovery cases and 2,203,677 total deaths since January 29,2021 . The numerous fresh cases stated daily rising rapidly, forcing administrations, and higher official authorities around the world to put in place a non-stop closure to ensure social segregation of the disease. The international reactions towards the disruptive distribution of COVID-19 have been speedily when most of the overstated countryside has blocked their corresponding borders without travel and transportation facilities. 
The WHO and CDC (Center for Disease Control and Prevention) distributed some formal guiding procedures ensured by the people, governments, nationwide, and worldwide organizations to guarantee the completion to prevent the disease, thus chain contravention promotes this outbreak epidemic [2]. The worldwide contribution for outburst COVID-19 as developed through the WHO including five major concerns: (1) to promote every aspect of individual health to maintain cleanliness and public awareness with social distancing, (2) managing rare or irregular cases to avoid society spreading, (3) to suppress the public communication by implementing appropriate limits, (4) relevance to decrease the mortality factor, some of the health-care services applied, and (5) the invention of developing of remedies and vaccines for major scale management. Figure 1 depicts the spreading of outbreak COVID-19.

The WHO and CDC panel has recognized with comprised symptoms showing a mild infection of COVID-19 as fever, dry cough, vomiting, diarrhea, and myalgia. The wide-ranging community has been knowledgeable that they will be quick to respond by seeking medical attention to reduce the incidence of illness. The government and official authorities have commenced investing more willingly in the COVID-19 research and its vaccine. In addition to this incentive program, there is a lot of research and development work directed at the COVID-19 epidemic.

The approaches, ML (Machine Learning) with DL play a significant part in diagnosing various diseases [3]. The method of image processing has increased considerable impetus in all areas of health care, particularly for the recognition of cancer in smart cities [4]. The current revision focuses on the DL and image processing contributions treated to battle the COVID-19 introducing an in-depth evaluation of the modern structure designed by using this technology.

\subsection{State-of-the-Art and Participations}

With the high effort headed for battling the coronavirus outbreak, scientific research has begun into every guideline, and DL combined among medical imaging practices has also been extensively tested to discover a specific resolution [5]. Many exploring studies have been available for related purposes, as described in Table 1 . The exclusivity of the current project performs in its efforts to highlight the medical imaging and DL approaches intended for detection

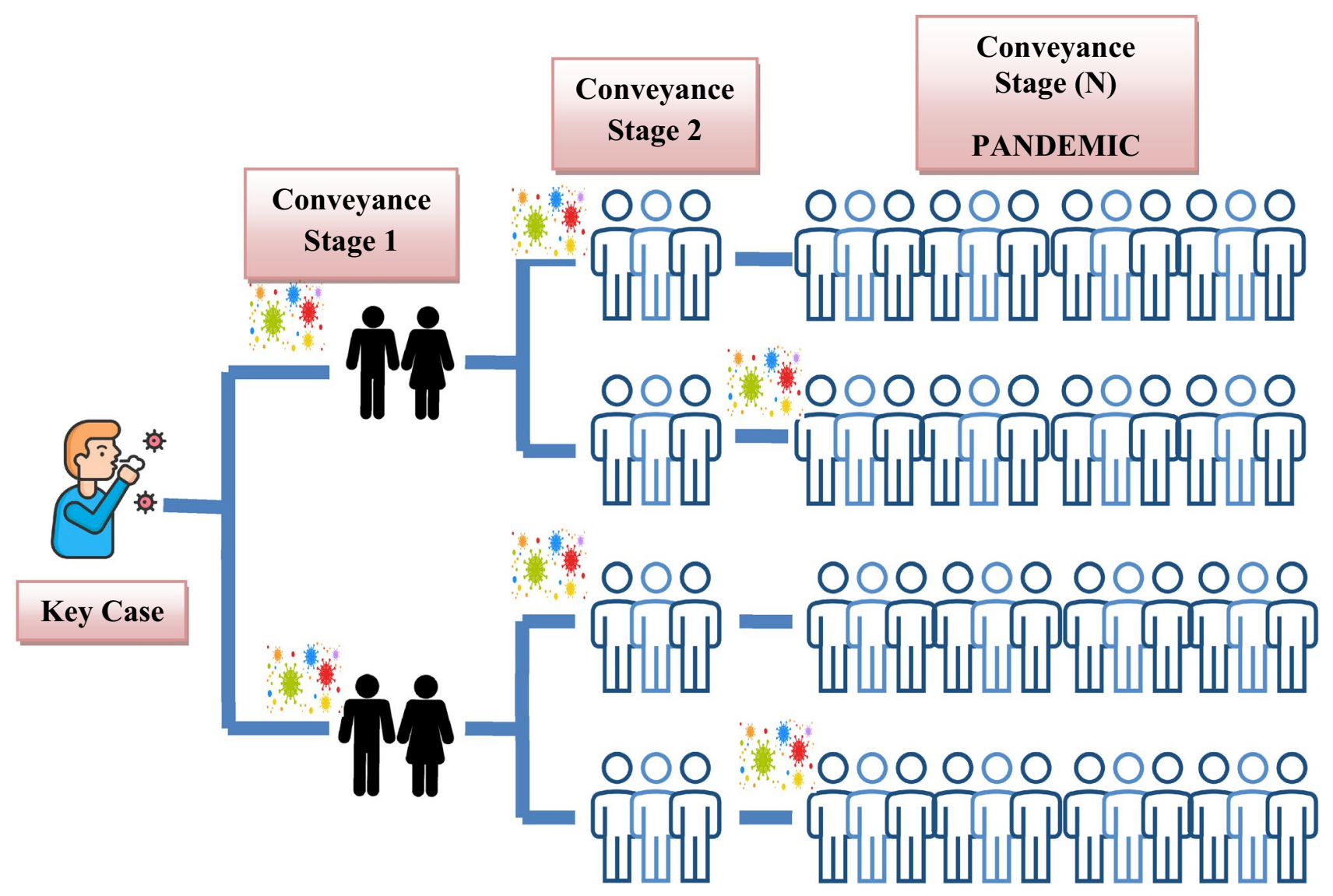

Fig. 1 Spreading of outbreak COVID-19 


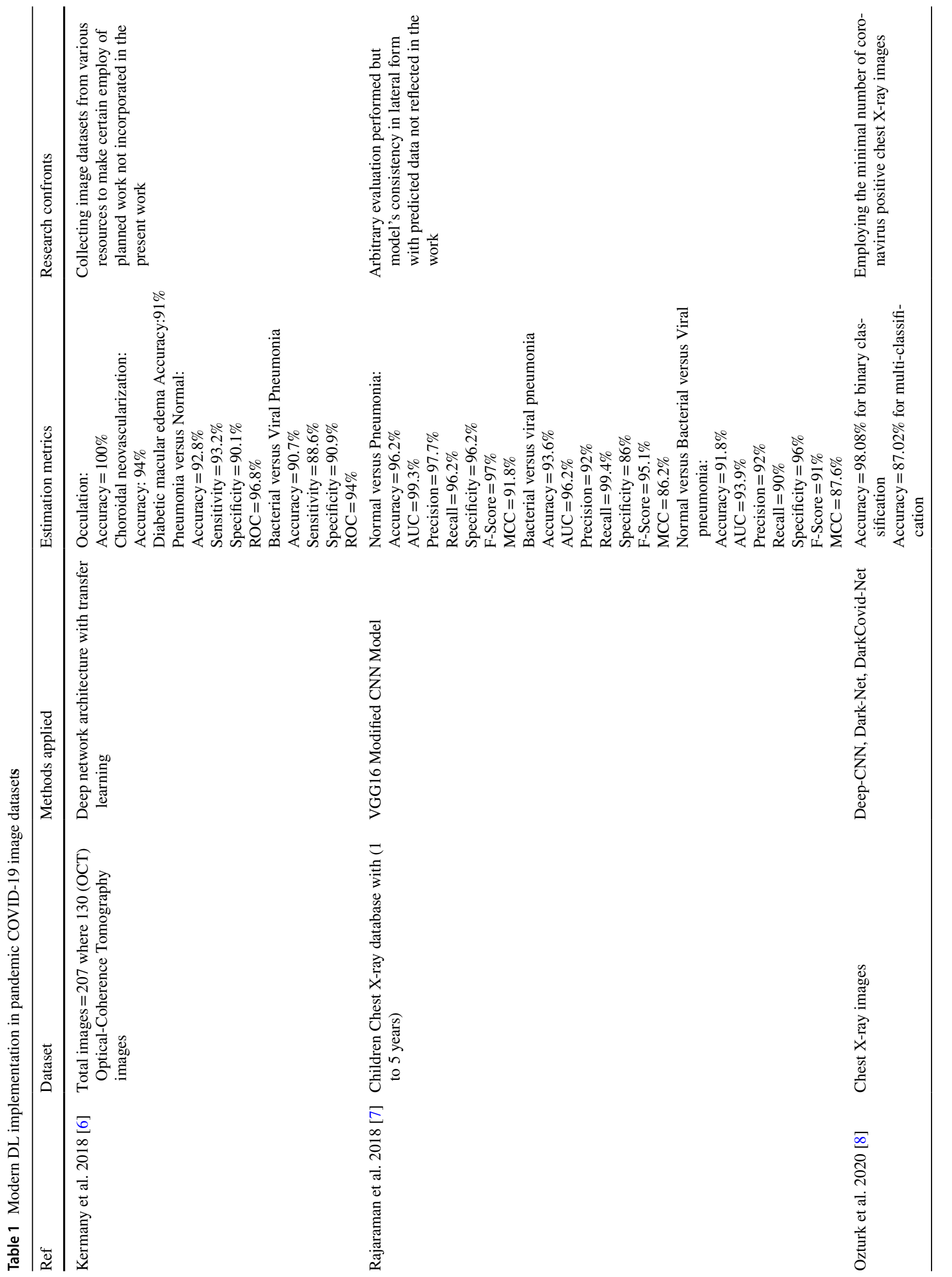




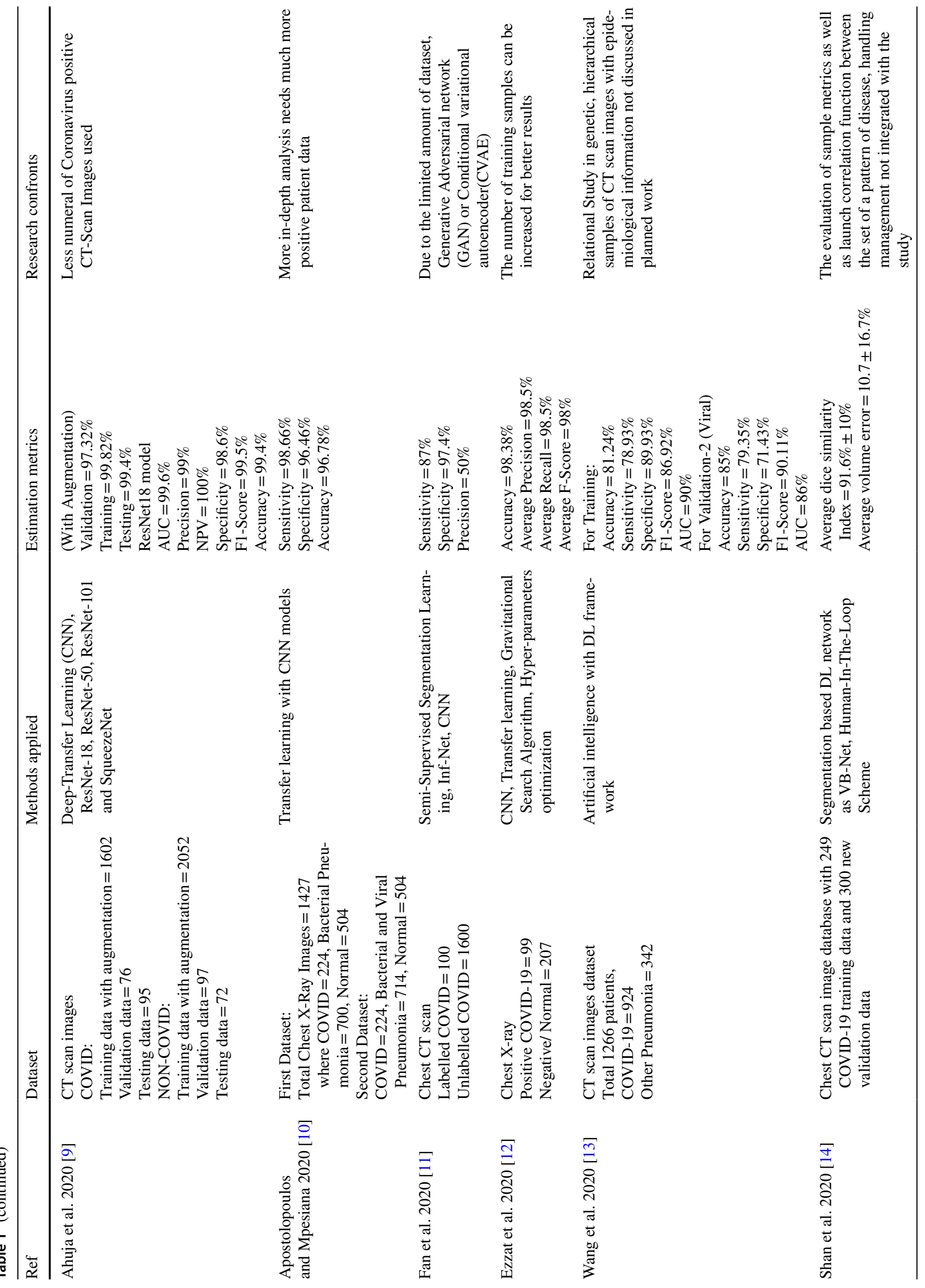


of COVID-19 as well as also gives importance towards the scope of the potential investigation, which still needs to be considered. The techniques extracted are based on various published articles by well-known publishers and therefore help to obtain critical recommendations from the research community and the management authorities against the disease.

The main problems in the COVID-19 study are the shortage of reliable and sufficient data. Because of the limit of tests performed, various death cases affected by the virus were left unreported. It's even harder to tell if something that fails in identifying COVID-19 illness is four, four hundred, or extra more. Globally, no country has been able to provide consistent data on the availability of the ailments in a sample representing the inhabitants. However, R\&D (Research and Development) work is unstoppable, so the integration of information plays a very imperative task. The scientific characterization of information integration depends on the quality of content, stated that "it is the main procedure of compiling and collating the information from several foundations that give meaningful information for the acquisition, detection, and localization of a particular object". In the scheme of ML with deep network architecture, the accessibility of huge, premium datasets achieves a chief task in the efficiency of the consequences. Data integration assists in compiling the compound data sets, in addition, to apply them to the DL framework for accomplishing improved correctness in prediction.

For instance, CT scan (Computer Tomography) medical images taken from the affiliated hospitals, named as Xi'an Jiaotong University with Nanchang Hospital as well as Xi'an No.8 Hospitals covers integrated part of the information compiling with artificial intelligence and deep network arrangement replica [13]. Another related work within the sequence of data integration where lung X-ray images were employed from Dr. Joseph Cohen's GitHub through added chest X-ray medical images found in Kaggle as a public database repository.

Ahuja et al. 2020 [9] defined the proposed work that COVID-19 diagnosis by using a three-phase discovery model as Stage 1-Data augmentation strategy with stationary wavelet technique, Stage 2-Diagnosis of coronavirus with prior-trained CNN framework, and Stage 3Irregularity localization in lung CT scans. The main criteria focused on previously trained structures such as ResNet18, ResNet50, ResNet101, and SqueezeNet meant for further investigational analysis. The dataset has 349 COVID-19 infected CT scan medical images out of 216 patients with 397 Non-COVID patients. There are $70 \%$ images and $30 \%$ images used to train the model and validate the system respectively. The investigational estimation performed with results that RestNet18 pre-trained transfer learning-based architecture will give enhanced efficiency of classification 
with the outcomes as $99.82 \%$ training, $97.32 \%$ validation, and $99.4 \%$ testing.

Apostolopoulos and Mpesiana [10] worked on X-rays with a transfer learning approach to identify coronavirus abnormalities in the medical images. The dataset consists of two sets as the first one, a total of 1427 chest X-rays with 224 coronavirus positive ones, 700 common-bacterial pneumonia images, and 504 normal images from Cohen, RSNA (Radiology Society of North America), and SIRM (Society of Medical and Interventional Radiology). In the new segment of the content, related references can be used for data integration to compensate for lack of information accessibility and proceed with generating improved quality prediction.

Ezzat et al. [12] presented a new approach called gravitational search optimization with the DenseNet121 CNN deep network model to achieve high accuracy in the diagnosis of outbreak coronavirus from numerous lung X-rays. The training with validation sets was introduced to evaluate the best possible value of hyper-parameters of the DenseNet121 model. Then, overall trained DenseNet121 is estimated using the test set. The proposed work is compared with several methods and reaches $98.38 \%$ accuracy for the detection of COVID-19.

Brinati et al. [16] Several studies engaged for developing some ML learning models to provide an identical reason by using minimum resources which resulting in considerably equivalent $82-86 \%$ accuracy, $92-95 \%$ sensitivity like the typical RT-PCR test. Ozturk et al. [8] The radiological imaging can collaborate with the high use of ML and AI models with a more precise diagnosis of the COVID-19 at prior guaranteed accessibility of treatment. The DarkNet as the classifier is used for YOLO (You Only Look Once) real-time object recognition scheme. This model can be engaged via the cloud with assisting the radiologists in authenticate their early screening, identify and categorize COVID-19 cases as well as provide the principle for particular locality wherever there is a lack of expert radiologists owing to an irresistible number of infected individuals.

Fan et al. [11] A novel automated lung contamination COVID-19 segmentation approach from CT scans through a deep network called Inf-Net, by summing up the features from high-level layers with PPD (Parallel Partial Decoded), then produce a global map for further experiments. A semisupervised approach can be used to improve the efficiency of the learning model with high correctness.

$\mathrm{Hu}$ et al. [17] presented a weakly supervised DL approach for identifying and classifying the COVID-19 illness in CT scan images with diminishing the manual tagging of the $\mathrm{CT}$ scan images. We considered an open public dataset, accessed from the TCIA (The Cancer Imaging Archive) for training a deep neural network for the identification of disease.

Khan et al. [18] suggested a novel, CoroNet: deep $\mathrm{CNN}$ representation which is used to identify coronavirus contamination from chest $\mathrm{x}$-rays. This method established Xception prior-trained scheme on the dataset as ImageNet which gives $89.6 \%$ accuracy in a 4-class model and $95 \%$ accuracy in a 3-class model. Pereira et al. [19] focused on the classification approaches as multi-class classification and hierarchical classification with a re-sampling scheme for the sense of balance in the class distribution factor.

Narin et al. [20] proposed the detection of coronavirus outbreaks on CXR images with three-layered deep NN named ResNet50, Inception-V3, and InceptionResNetV2. In this scheme, we used a dataset from the GitHub open source Repository allocated by Dr. Joseph Cohen and the Kaggle repository as well. The end result attained using 5 fold crossvalidation, ResNet50 $=98 \%$ accuracy, Inception-V3 $=97 \%$ accuracy and Inception-ResNetV2 $=87 \%$ accuracy.

Panwar et al. [21] performed a binary classification method to diagnose the COVID-19 samples. A novel modified VGG pre-trained model is used to categorize the input images. In this study, Grad-CAM (Gradient Weighted Class Activation Mapping) technique is used for color visualization to assemble the proposed DL model more understandable.

$\mathrm{Ni}$ et al. [22] conducted a lesion segmentation, detection, and position DL technique. The proposed methodology was trained in 14,435 individuals with lung CT scans for diagnosis of COVID-19 infection. The study was performed on a non-overlapped dataset of 96 infected reports from three hospitals in China.

Arora et al. [23] performed pre-trained models i.e. MobileNet, XceptionNet, DenseNet, ResNet50, VGG, InceptionV3 on the benchmark CT scan dataset to diagnose the COVID-19. To achieve better results, a high-resolution pixel of CT scans used with a dense network in the preprocessing stage has been executed.

Aslan et al. [24] studied two DL models to detect the positive cases of COVID-19 by using a lung CT scan and X-ray images. The CNN pre-trained architectures such as AlexNet and BiLSTM (Bi-directional Long Short-Term Memories) layer, which gives the best results in disease identification.

Jangam et al. [25] proposed new heterogeneous stacked ensemble models such as VGG, ResNet101, DenseNet169, and Wide ResNet50-2. From every CNN pre-trained model, the additional elements of base classifiers were obtained by changing the fully connected layers. The proposed model achieved good results on five various datasets, comprising of lung CT scans and X-ray images. The selected images are used to train and validate the proposed model which yields a high recall and accuracy.

Kumar et al. [26] presented an ensemble model consisting of GoogleNet, EfficientNet, and XceptionNet used to classify the patients into COVID-19, pneumonia, tuberculosis, or healthy. This model is used to detect the infected cases at a very early stage on the chest X-ray images. The proposed 
methodology improves the generalization of the classifier for both the binary and multiclass datasets. Two standard datasets are used to calculate the statistical performance of the proposed model.

Liu et al. [27] conducted a two-way transfer learning method for the correct segmentation of COVID-19 in lung CT scan images. Two major inventions in this study, consist of efficient virus segmentation DL model, known as nCOVSegNet, and a new two-way CNN transfer learning approach. The proposed work directs towards resolving the low edge contrast and huge feature combinations by using the effective segmentation method. The two-way transfer learning approach is used to make the facts and train on the CT scan images with COVID-10 illness.

Oluwasanmi et al. [28] presented the transfer learning approach with determining the problem of an insufficient dataset and the significance of semi-supervised GAN (Generative Adversarial Network) for features extraction with the production of images. The advantage of the proposed methodology is the loss ratio between the real sample and the produced data sample.

Perumal et al. [29] proposed the CNN transfer learning technique with the Haralick feature extraction approach which concentrates on the region of interest to diagnose the COVID-19. The main aim of the proposed work is to detect the COVID-19 infection at the very earliest stage to control the transmission of disease. This study makes the fast prediction task and helps several medical radiologists.

Prakash et al. [30] conducted a variant SqueezeNet pretrained model with segmentation technique to improve the diagnostic behavior of the $\mathrm{CT}$ scans and X-ray images. The proposed method is used to train and validate with the feature extraction from the medical image dataset for binary and multi-classification.

Essentially, the main target to understand the COVID-19 is at the max out where presented medical amenities are plagued. Emergency branches, outpatient wards have been extended away from their normal ability to serve growing patients. In this situation, healthcare contributors require to build resolutions with little information.

The physical composition of COVID-19 begins with mild or no indications, but it quickly transforms its track to make patients more sensitive to the deadly effects of multiple organ failure. The overall aim is to diminish the sudden decline, early diagnose of the illness using restricted databases. There are custom-made laboratory tests such as, RT-PCR (Real-time Polymerase Chain Reaction Test) which is tested through nose and throat-swab with narrow sensitivity. With the huge amount of patients, RT-PCR re-agents get limited as well as specific laboratory equipment for conducting illness testing is unavoidable. Various AI with ML is an effective technique that allows for faster-improved decisionmaking outcomes.
This paper provides complete details of the different uses of DL techniques in the COVID-19 pandemic employing instantaneous and public available databases. The exclusive offering to our study are listed below:

1. The study defines some essential contributions on outbreak COVID-19 and its prevalence, establishing the motive and the requirement for rapid spreading disease forecasting which assists to confirm the presence of the illness in smart cities.

2. The main responsibility of DL purpose in medical imaging is detailed converse to sustain its potential in the calculation of the impact of COVID-19.

3. The current research on DL performance and employing image processing in COVID-19 are elaborated.

4. The use of datasets, methods, testing metrics, research confronts, and some lessons examined incorporated with modern research activities as well as future indicators of epidemic control in various smart cities.

\subsection{Article Organization}

The work is arranged systematically as discussed further. Section 2 represents the basic details of COVID-19, DL with articulates the wide-ranging motivation for embracing development and scrutinizing the medical images from the available surveys. In Sects. 3 and 4, the general idea of DL's applications in medical imaging and it's related summary is discussed. A review focusing on the potential use of DL functioning in favor of medical imaging practices in COVID-19 is presented in Sect. 5. Section 6 recapitulates the above review which emphasizes lessons and includes suggestions that guide the potential outlook for future research. This paper is accomplished with the conclusion in Sect. 7.

\section{Background and Motivation}

This section represents the basics of outburst COVID-19, DL approaches and dealing with the analysis of medical imaging from the existing survey.

\subsection{Outline and Condition of COVID-19 Occurrence}

Initially, most cases of pneumonia were registered in Wuhan city of the PRC (People's Republic of China) in late 2019 [31]. COVID-19 as a transmitted virus commenced by (SARS CoV-2) severe acute respiratory coronavirus-2, called under the class of beta-CoV (Beta-coronavirus). Explorations have revealed that the foundation of illness may have originated from the Huanan seafood market in Wuhan city, and finally, the PRC government officially announced 27 more cases in December 2019. According to various 
studies, researchers found that the virus was passed on from wild bats [32].

According to the response of WHO, an R\&D Plan has been developed to speed up the diagnosis, treatment of this illness. The main proposal aims towards improvement in collaboration between researchers as well as worldwide health experts, to increase the R\&D development process and build new parameters to gain the knowledge for improving standards in universal response. [33] On January 30, 2020, after the various suggestions of the Emergency Committee, the General Director WHO, has announced that the outbreak comprised a PHEIC (Public Health Emergency of International Concern).

International scientists met at WHO headquarters in Geneva on 11-12 February 2020 to evaluate the existing knowledge about the novel virus, to get agreement on some significant research queries that require to be responded quickly, and discover paths to work jointly to speed up and support research for limit this outbreak criteria.

Shereen et al. [34] assert that the limits of the spreading of the COVID-19 or growth of the viral infection rely on natural conditions, respiratory water droplets, hygiene, and proximal space. The researchers, Kampf et al. [35] have found that the distribution of COVID-19 is nominal in high temperature and moist regions. They also emphasize employing a steam treatment to reduce the risk of the pandemic. The inhaled steam passes through the respiratory glands towards the alveoli as well as performing for improvement in the level of oxygen.

The WHO situation report, arrived with a valuable plan on February 3, 2020, to fight COVID-19 [2], which supplies some procedures and rules for higher official authorities, doctors, etc. ensuring to fight with the pandemic as well as personal safety for frontier ones. An additional significant plan was provided by the WHO to perform lockdown worldwide, which could be effective to avoid infection. The principle reports generalized [36] by WHO on March 19, 2020, addresses the information of avoiding the infection, totally based on positive samples by severe acute respiratory syndrome infection (SARS).

The situation report also focused on the particular precautionary measures as the use of a mask by the community on COVID-19 exaggerated regions, hand washing with alcohol-based sanitizers and health workers instruct not to touch patients with uncovered hands. According to the WHO, there is no definite medicine for the illness extremely. The UNCTAD (United Nations Conference on Trade and Development) has compiled a list of best observations, strategies [37] that countries can use to implement Key provisions that can assist economic growth during the pandemic.

\subsection{Preliminaries of Deep Learning}

DL and (NN) Neural networks expand considerable impetus in modern scientific research by building the potential to gain learning from the context [38]. These methods have been extremely employed in different applications like object identification, image recognition, prediction, classification, smart vehicles, etc. owing to their ability to a wide variety of data in the different areas. Different techniques worked with the DL approach as shown in Fig. 2. The DL methods are used to imitate the human brain functionality in the preprocessed form of information with more precise results. The deep network has trained like a human mind works accordingly by employing different layers to help predict and classify the data.

The model learning with supervision, as trained in pairs of identified inputs/outputs. The already identified value creates a vector of input and output called supervised learning. The supervised classification techniques [39] were performed to predict the clinical decline factor, death rates in ICU, generalized the clinical predictions for complex sepsis prognosis.

The unsupervised learning model works with the relationship between classifying the data elements without getting the label information. Object detection, neural network, clustering approach, etc. techniques followed by different algorithms. The unsupervised deep network framework used for medical image classification [40]

Learning with a semi-supervised approach, consider an intermediate technique towards ML models. Labeled as well as non-labeled values for training statistics employ in the semi-supervised learning method. When non-labeled data value is combined with some labeled value, so we can get better accuracy with improvement. The deep network framework augmented by [41] where performs label learning for non-labeled data, classifying the brain tumor cells with improved accuracy.

Different DL practices use ANN (Artificial neural network) to extract the features accordingly. The approach of feed-forward by Xu et al. [42] is used to provide knowledge in which every level refreshed its input value to get results in the summarized form. Enhanced ANN technique deals with evolutionary models that can provide better results comparing with traditional methods.

RNN (Recurrent Neural Network) mechanisms are similar to convolution neural networks, except that RNN is employed for language estimate computations. RNN follows the feedback approach where the output of a single layer is fed the same as the subsequent layer input. Both CNN and RNN as hybrid optimized architecture as FC-CSO algorithm with AND operation which enhanced the hidden layers used to detect mammogram breast cancer accurately by Patil and Biradar [43] 


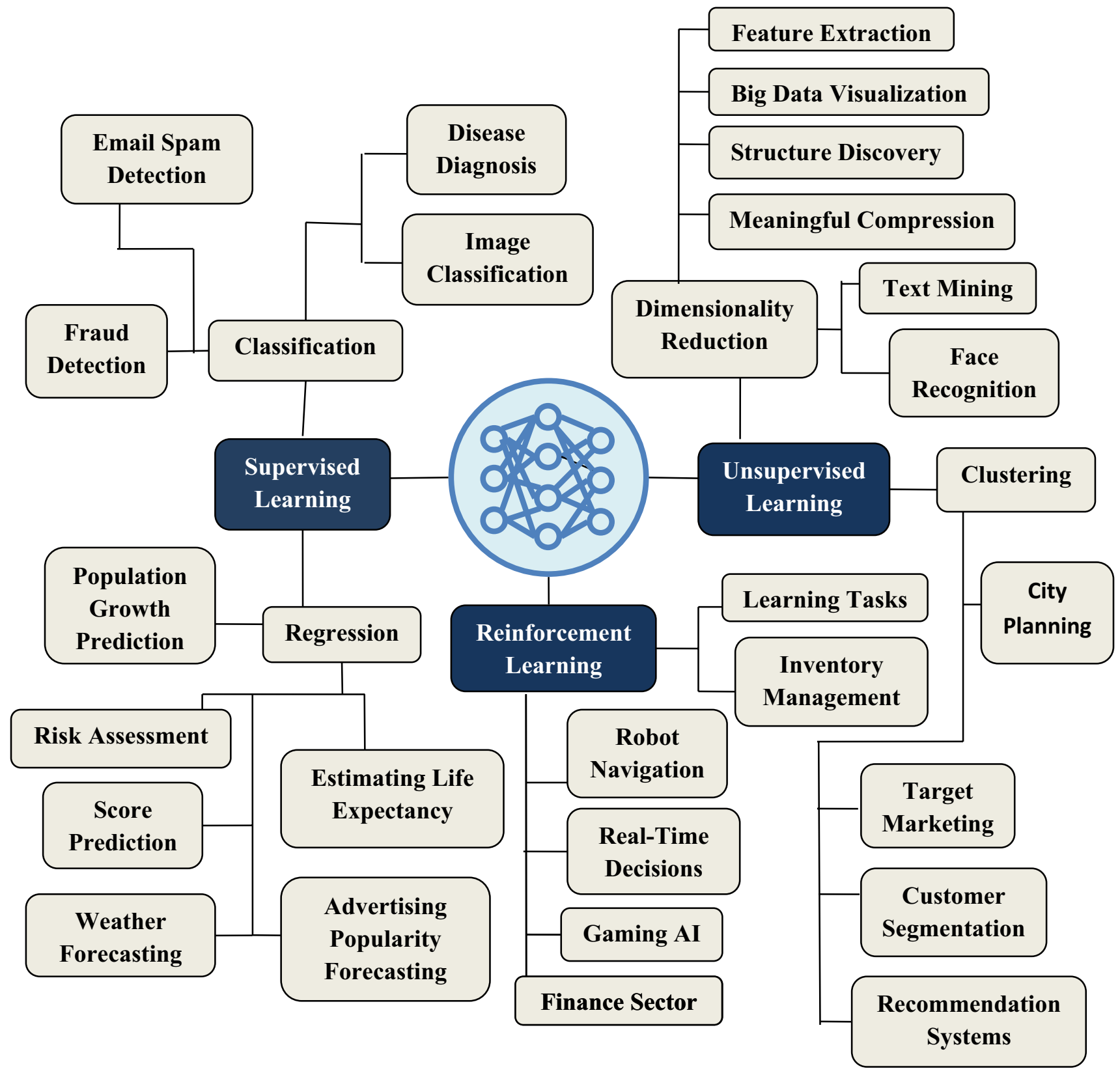

Fig. 2 Deep Learning applications and techniques

GAN employs the technique of discriminator and network system of generator. When the discriminator separates the false and factual information, then the generator system generates false information. To improve the training process, the deep network techniques were performed accordingly. As a result, GANs are extensively used in image processing techniques [44] as in the invention of images from text data. The network was introduced by Google, as an inauguration block for computing convolution as well as pooling tasks that concurrently process complex operations efficiently. Superior stage of DL used for automated computation in image processing [45]. For example, A novel method based on an in-depth synthetic image processing study where semantic extraction of the colony of bacteria's on agar plate images to amplify the training data set size [46].

DL plays a vital role in the implementation of some algorithms on a large dataset. DL has huge potential to cope up with large data samples that will be created in smart cities owing to digital integration [47]. DL strategic testing depends on two main parameters: Firstly, the large size of the dataset to be in practice, and secondly, the maximum computation superiority. DL also facilitates the rapid investigation 
of multifaceted medical images [44]. DL approach is broadly used for the sustainable expansion of smart cities through the use of mass surveillance [48]. The subsequent section supplies an outline of DL's techniques for medical imaging.

\section{Indication of DL Application in Medical Image Processing}

Different medical data sources with MRI (Magnetic Resonance Imaging), X-ray, CT Scan (Computerized Tomography), PET (Positron Emission Tomography) images, have offered doctors a huge amount of information [49]. The efficiency of ML algorithms focused on different aspects of feature extraction and data illustration. ML techniques are challenged by two factors, firstly, to get high accuracy with large dimensional image data samples and secondly, to train the model to get the most suitable results [50].

DL algorithms have been widely used for ensuring the uppermost level of correctness in form of illness detection and forecasting. The use of the DL procedure has brought about new developments in healthcare, as exposed in Fig. 3.

Litjens et al. [51] evaluated different DL approaches in medical imaging procedures with included several applications as Image Segmentation, Image Classification, Entity Identification, etc. In the discipline of medical imaging, the DL is used in several departments as Heart disease diagnosis, detection of cancerous cells, neurology, ophthalmology, psychotherapy. The creators are also entitled to some unsettled DL research challenges associated with image investigation. In the recent circumstances of affected people and another medical collaborator who preserve the electronic

\section{Diabetic Retinopathy}
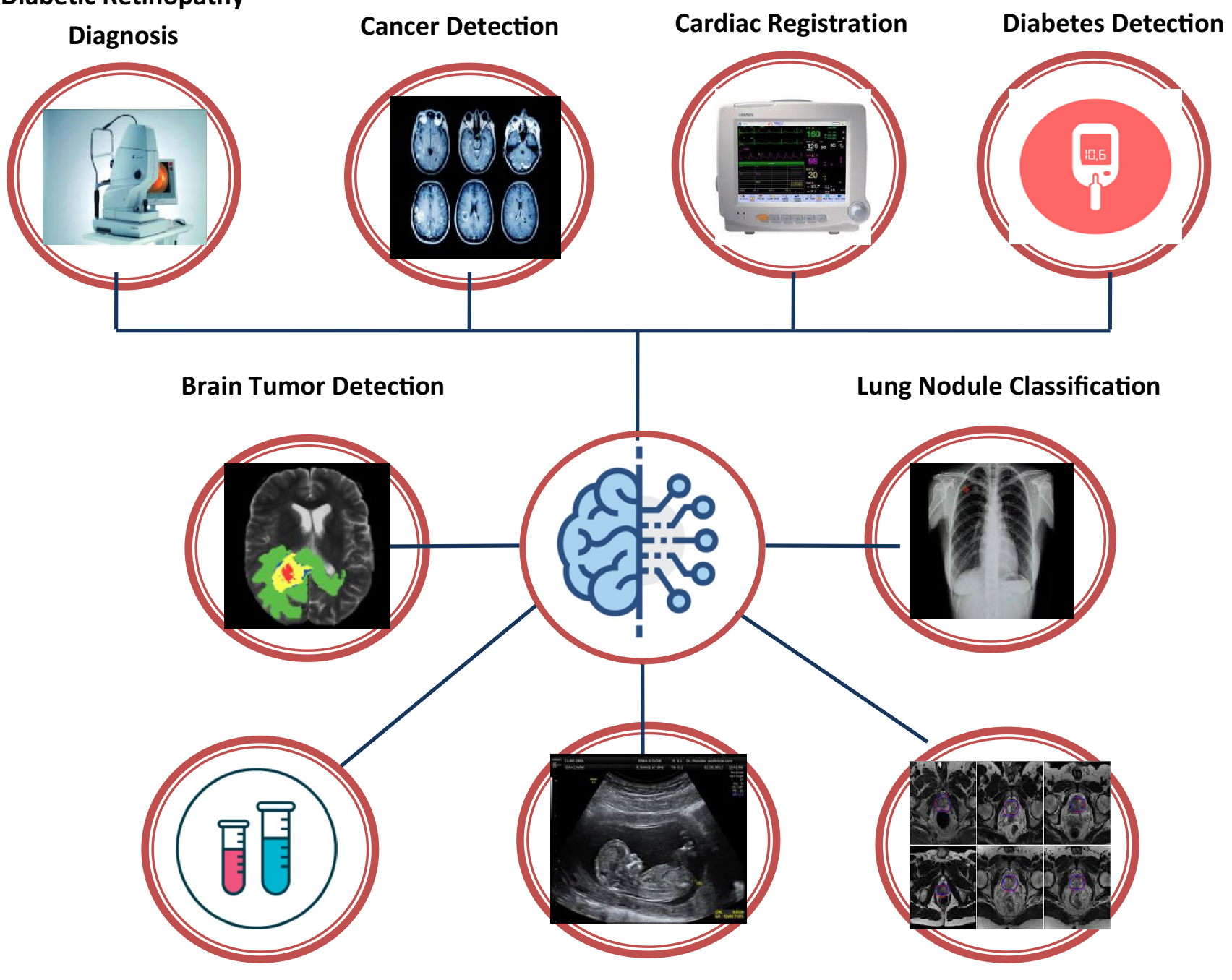

Thyroid Diagnosis

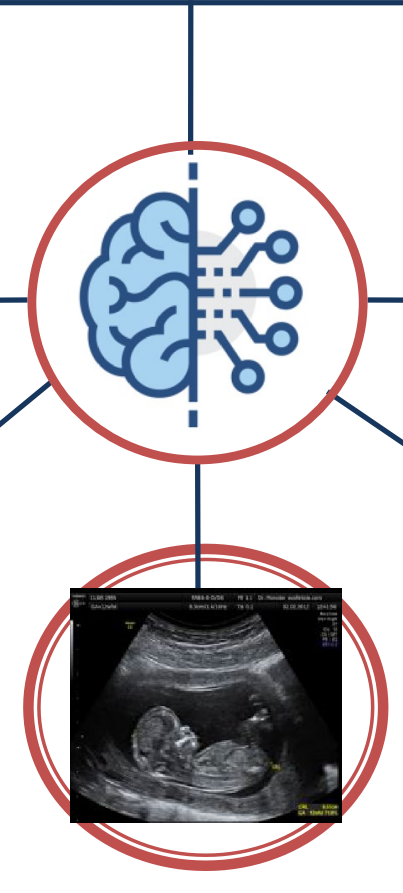

Fetal Localization
Lung Nodule Classification
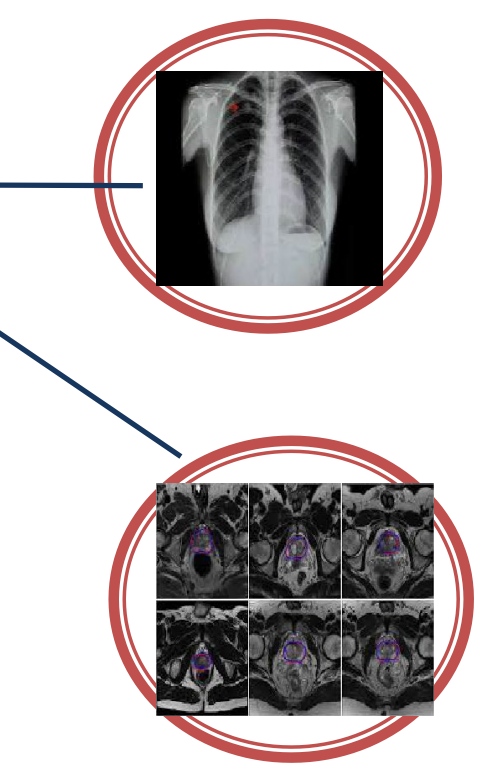

Prostate Image Segmentation

Fig. 3 Various Deep learning applications in Medical Image Processing 
documentations, AI approaches that help in reducing the processing of medical images.

Ker et al. [52] evaluated the different AI strategies that can be used in medical imaging analysis. They have found from different surveys that $\mathrm{CNN}$ is extensively used in image analysis with large data sample processing techniques. The researchers also emphasized the major challenges of the non-availability of high-definition data samples with a labeling approach for improved understanding.

In the recent research, deep $\mathrm{CNN}$ with stochastic pooling instead of max-pooling or average pooling, more efficient for the identification of COVID-19 in the chest CT scans due to examine the features from the data samples throughout the training scheme and not required too much time for feature production [53].

\subsection{Image registration}

Image registration is a way of translating the data sets into a solitary coordinate replica. Registration performs a significant task in the field of biomedical image processing. It is widely used for data analysis or data joining together from various medical foundations. Normally, a medicinal professional should show various images in a variety of ways to minimize the illustration contrast in between the images [54].

The medicinal professional is frequently predicted to physically categorize the point in the images with important signal variability as being part of a large framework scheme. Biomedical image registration accumulates time-consuming tasks for physicists and doctors. To concentrate on the problem of the labor-intensive image registration system, the use of DL in this process has enhanced the efficiency of the dedicated image registration procedures [55].

Above $50 \%$ of cancer-affected individuals receive radiation rehabilitation, producing it as an individual most ordinary cancer healing scheme [56]. While the integral numerous affected patients increases, a large number of doctors will help out with medical imaging techniques. The open fully mechanical 3D registration software as 'Elastix', which improves the radiotherapy progression in radiation-oncology branches globally. The planned representation offers a computerized search, which works well internationally. Formerly, it was so hard to find three-dimensional image registrations that can provide accommodations with several changes towards the position, translation, and dynamic intensity variation efficiently. The main purpose of the proposed scheme is to save time prominently for physicians and provides high-quality clinical enrollment results. Improved ability to integrate diagnostic scanners which ensures the affected patients collect fewer repetitive scans and reduces the radiation exposure. The investigational result of the Haukeland Medical Center cancer database found a 97\% success rate of prostrate, $93 \%$ of seminal vesicles, as well as $87 \%$ of lymph joints.

Chee and $\mathrm{Wu}$ [57] employed a self-supervised learning approach with a neural network to set up three-dimensional registration schemes. The main purpose of this mechanism is to facilitate image registration in a short period of an instance to identify the interior regions of the brain by capturing the data from various statistic categories. The tentative outcomes performed based on the orthogonal view of the brain scanning were found as $100 \times$ more rapidly running time.

\subsection{Segmentation}

In medical image practices, Image segmentation performs an important task in the diagnosis of illness. Image segmentation splits the required image into various segments which aims to examine the digital images faster and simpler.

The novelists Oktay et al. [58] proposed a new method to conquer the disputes using a standard training approach that incorporates previous knowledge into $\mathrm{CNN}$ with a welldefined trained standard model. The recognition of biomarkers into medical images assists in the diagnosis progression. The supervised DL method is widely utilized for imaging practices but cannot be accurate because it requires in-depth knowledge of organs through their positional characteristics. The biomarkers detection procedure can be obtained by distinguishing unfavorable areas. This kind of incongruity can provide unseen information regarding structural formation.

Biomedical image segmentation is a compilation of some dedicated medical fragments that cover the entire medical digital depiction [59]. Recently, most interdisciplinary methods are being employed to process the medical data to obtain better diagnostic accuracy.

Guo et al. [60] proposed a monitored approach as a supervised ANN technique through the integration of cross-modality features where used at all levels of the ANN model. Besides, the authors also discussed a method of image segmentation by using CNN to describe the soft tissue lesions in images attained by different disciplinary procedures such as CT scans, X-rays, PET, MRI images. Appropriate mining with feature exploration of the tissue is necessary before image practices. It preserves to be helpful in situations wherever the medical images are uncertain or else have errors throughout the organization breakdown. A major challenge to existing medical imaging techniques is the deficiency of synchronization between training objects and output dependence.

The authors, Seebock et al. [61] perform the concept to apply the Bayesian DL process assumes that pandemic uncertainties will be related to framework manipulation from the trained database. The researchers also use the BayesianU-Net to training based on the condition with weak anatomy 
labels provided using various ANN techniques. The MRI images of the prostate by using segmentation can reveal important details for detecting prostate cancer. However, there are many challenges towards MRI image segmentation as loss of boundary area between the prostate gland and other organs, multi-complicated areas, and differences in the prostate features.

\subsection{Image Classification}

The classification is recurrently called CAD (ComputerAided Diagnosis) performs a significant part in medical imaging tasks. Throughout the classification procedure, one or more images are considered input data samples and produce one diagnostic sample as an output that classifies the image accordingly.

Rajpurkar et al. [62] presented a novel "CheXNET" DL model that includes 121 convolutional stages, 1,21,120 chest $\mathrm{X}$-rays offered as input data for detection of 14 various types of lung diseases. By examining this test, the radiologists claimed that the CheXNet mechanism goes beyond the F1-metric efficiency range.

Mikolajczyk and Michal [63] presented a study that deals with the problem that arises in the field of ML, the shortage of adequate quantity of training data or irregular class equilibrium within the databases. To overcome this problem, data augmentation is used, where the task of image classification begins with standard image transforming techniques as cropping, rotating with an angle, zooming, histogram mechanisms, and concluding with GAN examples.

Shankar et al. [64] proposed a novel automated HPTIv4 (Hyperparameter Tuning Inception-V4) scheme for the detection and classification the diabetic retinopathy from colored fundus images. At the initial stage of preprocessing, CLAHE was used to improve the contrast level of the fundus images, and then a histogram-based segmentation approach was performed for attaining segmented images. The HPTI-v4 representation is applied for extracting the features and performs classification using MLP (Multilayered Perceptron) method. This proposed model will give better results with $99.49 \%$ accuracy, $98.83 \%$ sensitivity, and $99.68 \%$ specificity.

Raj et al. [65] presented an advanced classifier as optimal DL for diagnosis of lung cancer, brain imaging as well as Alzheimer's disease. The main purpose of this research is to find a model to select the appropriate aspect of medical image classification. The enhanced classifier as OCS (Opposition-based Crow Search) method is performed to select the features from dedicated images for further analysis. This proposed model will give the maximum efficiency with $95.22 \%$ accuracy, $86.45 \%$ sensitivity, and $100 \%$ specificity for the set of images used.

\subsection{Image Localization}

In the image classification process, the processed images are supplied to the $\mathrm{CNN}$ model, and then the medical image content is visible. After the image classification, image localization can be used for the detection of illness. It is mainly reliable for introducing the bounding box in the region of an output location, which is also termed as classification employing the Image localization approach. The image localization of any structural part is a necessary preprocessing part in the scientific identification that allows the radiologists to identify certain significant features. In recent years, various research works encompass conducted the mechanism by using DL models to focus on the localization of the illness.

Guo et al. [66] presented a model for obtaining T2 MR prostate images to locate it accordingly. There are several challenges to classify accurate prostate in images as differences in thickness and incompatible manifestation. The authors performed the SSAE (Stacked Sparse Auto-Encoder) for training the prostate cancer images and further instructed characteristics to reveal the prostate identity in the medical images. This work has been executed on a database including 66 prostate MR images with accurate findings that will provide better results as compared to existing methods.

Baumgartner et al. [67] produced a representation that assists in the localization of fetuses in the images. Throughout the procedure, CNN performed training to classify 12-scan test planes with the network framework intended to identify the fetal effectively. Tentative results accomplished with recall $=80 \%$, accuracy $=81 \%$ and precision $=69 \%$.

Shin et al. [68] proposed a framework for mass localization and classification in breast ultrasound images. The study found that training CNN-based detection with huge and weakly annotated databases performed a non-trivial dilemma, and then over-fitting that can be occurred in those training with little and strongly defined database. To overcome this confront related to over-fitting, we use a database with weak annotations and a small one strongly defined database in the form of a hybrid. The test outcomes signified that the projected model may perform effectual localization and divide the masses with a little annotation effort. The experimental results were trained with 10 robustly images with weakly annotated images were matched to trained results from 800 strongly defined images, with confidence level 95\% in difference with time -3.00 to $5.00 \%$ in the principles of appropriate local measurement (CorLoc), a scale of images with ground-truth is higher than 0.5 .

\subsection{Detection}

$\mathrm{Xu}$ et al. [69] planned a DL representation model named SSAE (Stacked Sparse Auto-Encoder) to detect the nuclei 
in the medical breast images. The SSAE trained to extract the superior features, and extruded characteristics are considered input into the 'softmax' detector for detecting the nuclei in the Images. Test results claimed that the proposed model attained $78.83 \%$ accuracy and $84.49 \%$ F-Measure.

With the latest advances in DL mechanisms and computer-vision methodology, medical imaging applications are free to expand as never previously. Object recognition is a necessary part of the computer vision method to identify real-world objects. The object detection technique permits the correct identification with localization of several entities in a dedicated digital image or video. This technique is used to train the prognosis models or use a similar pattern to identify and locate the objects. Object detection approach is a major technology used in various applications as image retrieval mechanism, medical diagnosis system, video surveillance [70].

Ghesu et al. [50] planned a DL model with a marginal space approach for object detection. Flexible training models are used to obtain better efficiency in deep neural network layers. The estimated positional range, boundary demarcation, integrated with the DL model and discover image delineate segmentation. The investigational methods included 869 patients, images with 2891 aortic-valve, distributed $45.2 \%$ enhanced efficiency as compared to the other preceding methodologies.

Other interesting research, Shin et al. [71] presented an original approach by training the $\mathrm{CNN}$ model with the Triple cross-validation method to diagnose the central lung infection, lymph nodules. Test results reach $0.95 \%$ AUC, 85\% sensitivity. Appropriate with GPU (Graphics Processing Unit) memory limitations, improving 2D image recognition is a major confront in object detection mechanisms. An additional fascinating work, Liao et al. [72] has proposed a novel method for detecting lung cancer. This mechanism includes two necessary steps: firstly; to detect uncertain pulmonary nodules by performing 3D NN, secondly; to diagnose cancer by collecting five fine nodules and attaching them to a Leaky-Noisy OR gate. The experimental outcomes were obtained with accuracy $=85.96 \%$ in trained datasets and $81.42 \%$ in tested datasets. Perceiving an accurate ML model technique that can differentiate, locate, and detecting the multiple object entities in a solitary Image left with a major confrontation in the computer vision [73].

\section{Summary}

In the above sectional part, the studies have discussed DL's applications in medical imaging mechanisms as image registration, segmentation, classification, localization, and detection. The recent advances in DL's progression, seen major transforms in health care by introducing new openings to improve people's lives. DL for biological-image analysis is widely employed in the technical controlled field to locate and classify different types of diseases. According to the survey, it is analyzed that the DL models perform well in many applications related to medical imaging. Earlier, doctors had spent enormous time in reporting, and many targets have done manually. DL used to recover its time-consuming procedures and obtained better results over the past experiences, offering enhanced services with classic medicalrelated tools. The medical imaging practices with DL build the healthcare organization by offering new opportunities for enhancing medical health for society. The studies summed up the presented DL's applications into medical image methods as shown in Table 2.

\section{An In-depth Study of DL in Favor of Medical Image Processing in the Outbreak of COVID-19}

This segment of paper converses the latent of DL in the medical imaging practices to fight against the COVID-19 by using four precious tasks. The approaches are outburst prediction, the coronavirus transmitted indication, detection and therapeutic aspects of COVID-19, vaccine availability, and remedy research as depicts in Fig. 4.

The X-ray images are used to detect pneumonia and the underlying cancer stages. But CT scan images are a more extensively and grown-up method that can be intended to identify microscopic changes in the internal structure of organs. The X-ray images can be unsuccessful in recognizing the soft tissue-related diagnosis as they use the 2D imaging method but alternatively, CT Scans use 3D computer imaging technology as the scanning takes many images from different parts of the human body. Even-though, both CT scans and X-ray images encapsulate Images of interior body composition, as in the existing X-ray approach, images are often. For example, the ribs cover the heart, lungs building the structure of large medical questions to be hidden, thus deteriorating in terms of highly accurate diagnosis results. On the divergent end, in the event of CT scans, the overlapping behavior is entirely removed to make certain the interior composition is more transparent and grants a clearer sympathetic of health conditions.

Early detection of pandemic COVID-19 illness is often based on the vital indication of pneumonia, a patient's tour history, and contact with another infected individual with COVID-19. Except that chest CT-scan or X-rays performs a significant task in recognizing the magnitude of the infection and proceeding needs. The indications of COVID-19 infection usually have inconsistent or subtle dense airspace irregularity. The chest CT scans give indications about two-prolonged lung participation. The COVID-19 infected 


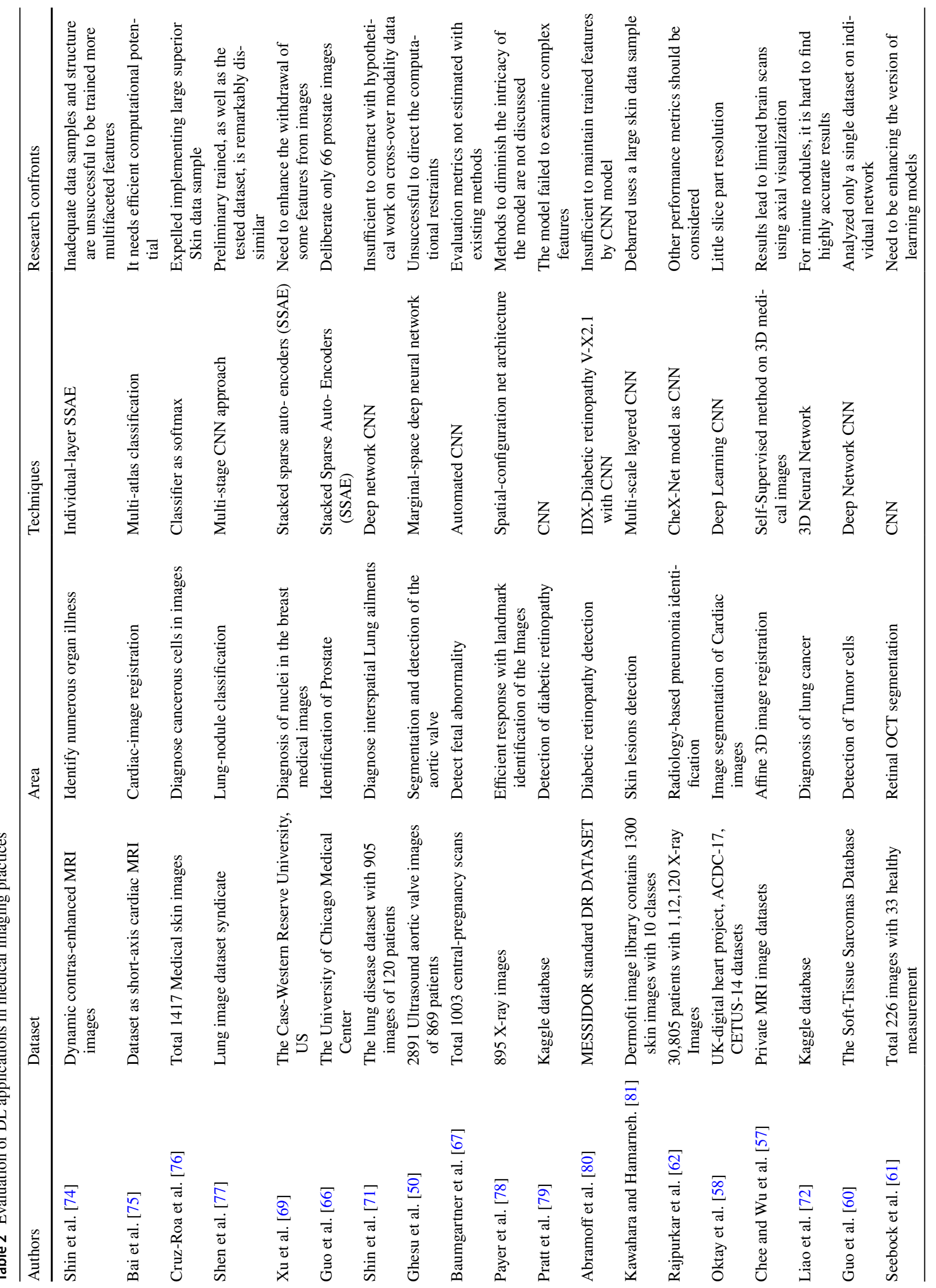




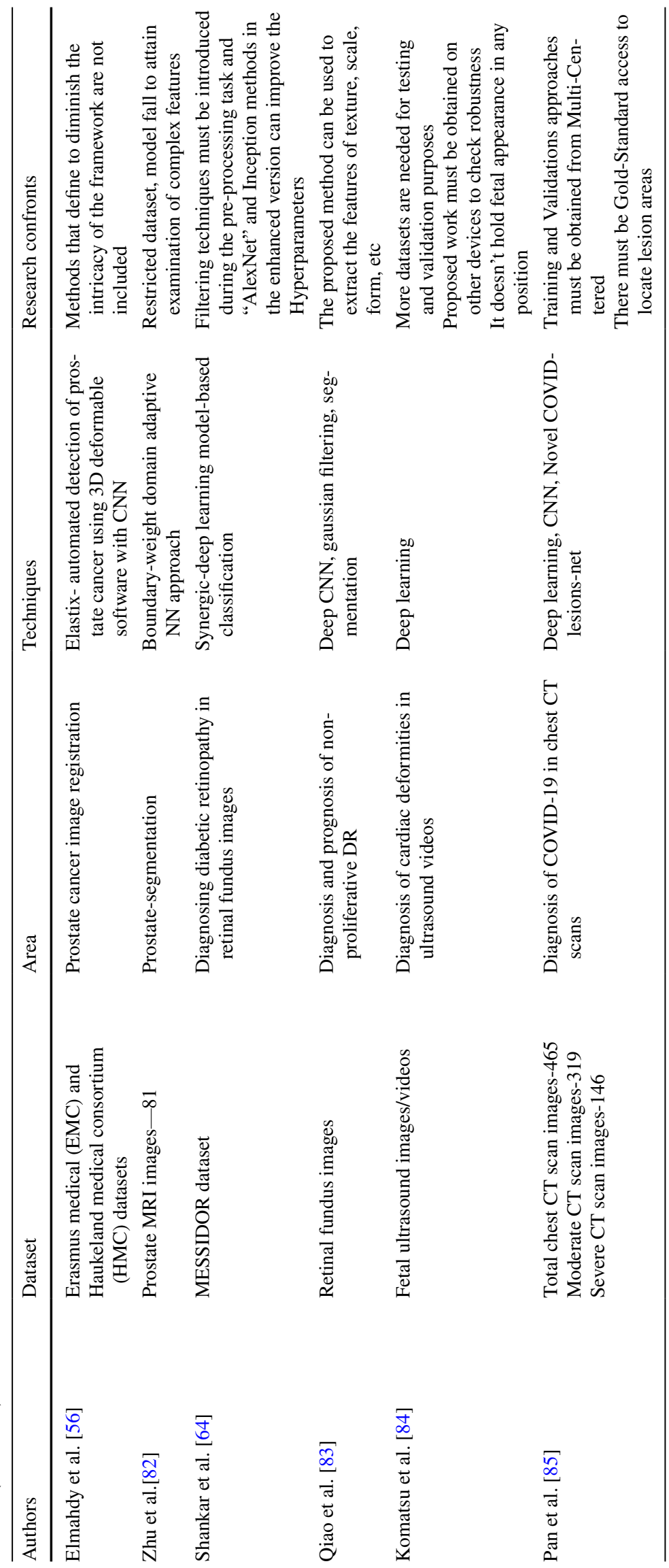


COVID-19 Medical

Image Dataset Resource

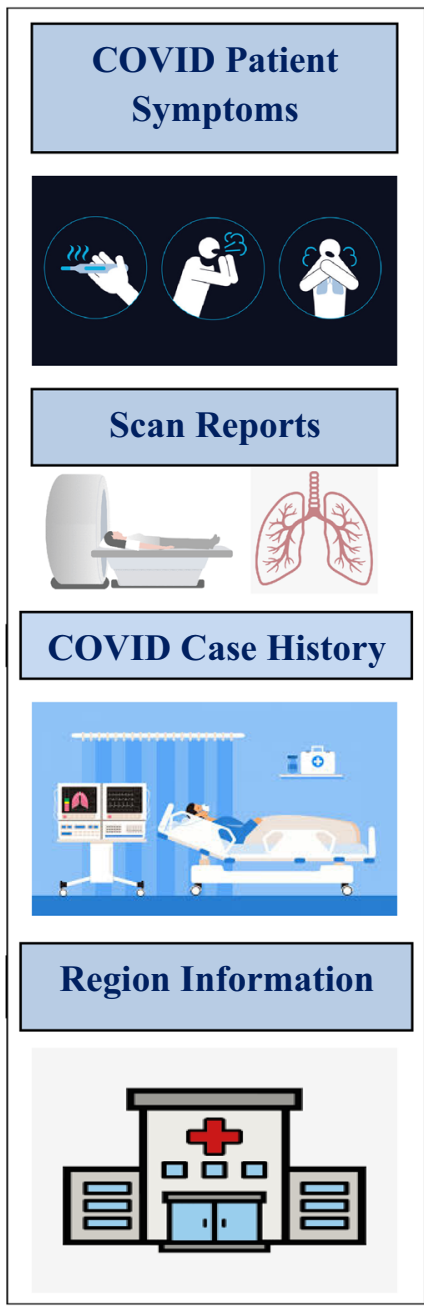

Deep Learning

Applications

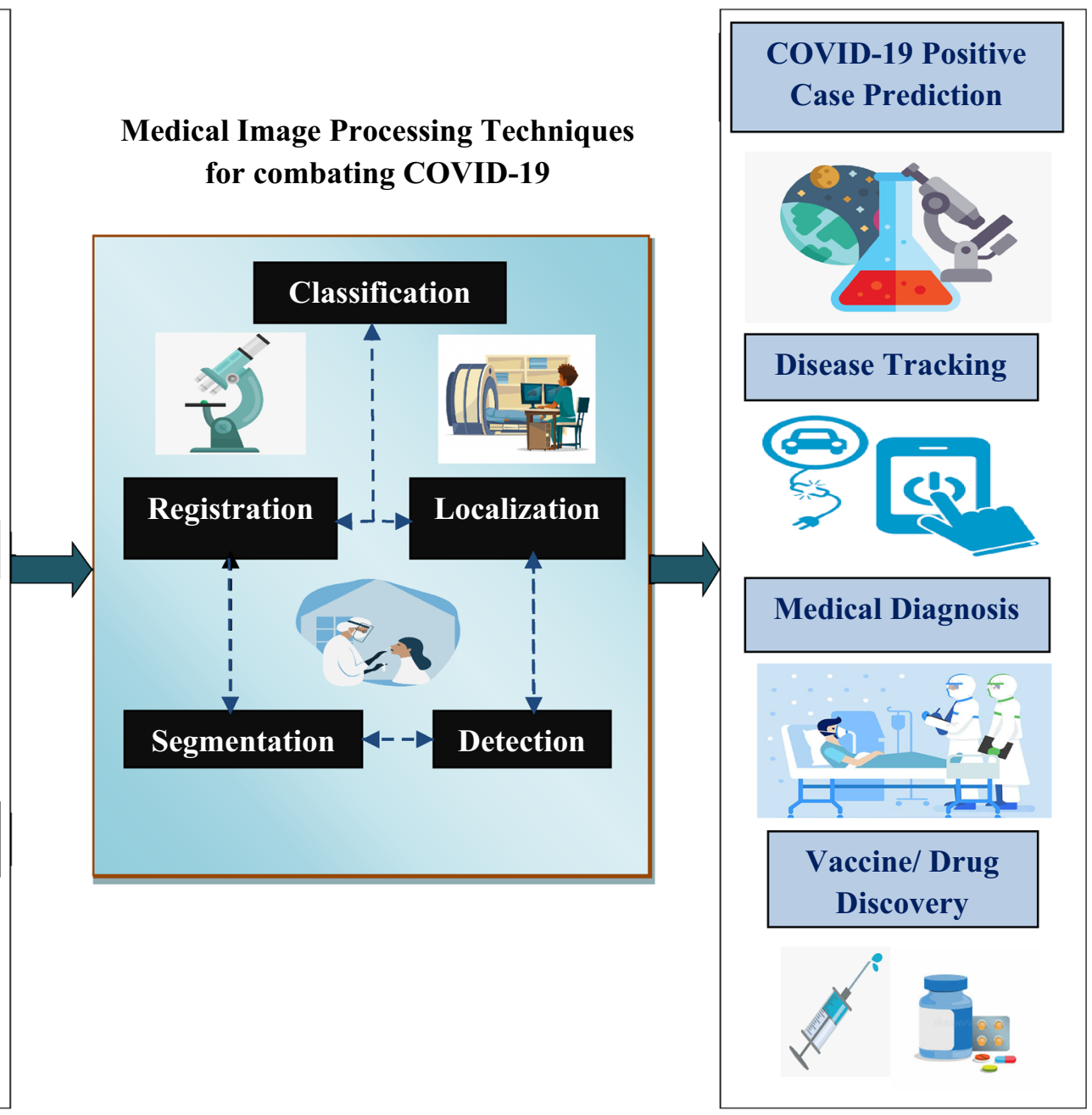

Fig. 4 Deep Learning practices in Medical Image Processing techniques to battle with COVID-19 Epidemic [86]

patients with critical circumstances in the ICU showed a coherent pattern, while the patients are non-ICU showed a wide pattern with grounded glass in their official reports. In contrast, chest medical images having SARS-CoV and MERS-CoV illnesses have shown one-sided indicators. However, in $15 \%$ of infected cases, the starting chest X-ray images showed the normal range of patients already contaminated. This highlights the need for additional validation by physical examination or through DL mechanisms.

In the lung, CT scans and X-ray images are captured of a specific segment, along with rotation from diverse angles. The images are accommodated on the computer and are further processed to build a new original image that removes all overlapped traces. It assists physicians to understand the improved interior structure with enhanced clarity to get some absolute design of compactness, shape, surface consistency, and parallel outline. Moreover, CT scans are highly considered more effective than other X-rays. The CT scans or $\mathrm{X}$-ray images failed to distinguish between viral pneumonia as coronavirus and other bacterial pneumonia as common cold-related indications. These images are widely used to detect the occurrence of the illness, which can also be the result of any other ailments. Also, the coronavirus disease is highly contagious, and the use of medical imaging tools worked on many infected patients is extremely dangerous. The scanning machines are extremely complicated equipment, and make cleaning the pieces of machinery each time after an individual patient practice is not possible. Despite numerous attempts to clean it up, the high risk of infection from the scanning machine equipment area is at its peak. In contrast, the swab trials have proven to be highly efficient in the diagnosis of COVID-19 than imaging mechanisms. 
Some COVID-19 infected patients have typical normal CT scans or X-rays but have been diagnosed later with a positive one. The conventional mechanism RT-PCR can diagnose the illness with high accuracy but has the corresponding confronts of time-consuming to detect and need of several reagents. In this time of health crisis with highly virus spreading rate, the greatest need is to quickly diagnose the disease with limited resources. To meet this requirement, ML-based algorithms with medical imaging techniques perform a vital task in eradicating the irresistible crowd of accomplishing the swab experiments and other relevant ones. The sample medical images as CT scans and X-rays of COVID-19 individuals are shown in Figs. 5 and 6.

\subsection{Outburst Prediction}

The globe is facing an exceptional universal health disaster owing to the COVID-19 pandemic [89, 90] DL techniques with medical image practices, have been found extensively together and its related works [91, 92]. As it has already become a trending method surrounded by researchers in the field of healthcare departments, it is logically recommended as a suitable model for current outbursts. For example, datafocused studies as DL models, Computer vision, medical image processing has proven effective in enhancing their appropriate extremely favorable solutions [93].

DL makes COVID-19 predictions worldwide accordingly. The efficiency of COVID-19 may be depending on the numerous factors classified as (1) Confirmed; (2) Active; (3) Deceased; (4) Recovered; (5) Population; (6) All reported dates; (7) Locations; (8) Living Circumstances, etc. The DL approach also produces data-oriented features as well as supervises the high-quality data, while ML mechanisms focused on handmade materials and compare with fewer quality data samples [94].

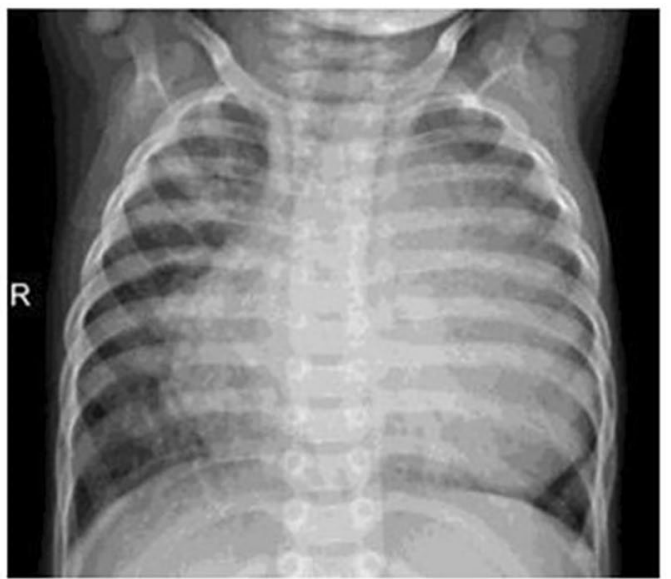

(b) Bacterial Pneumonia

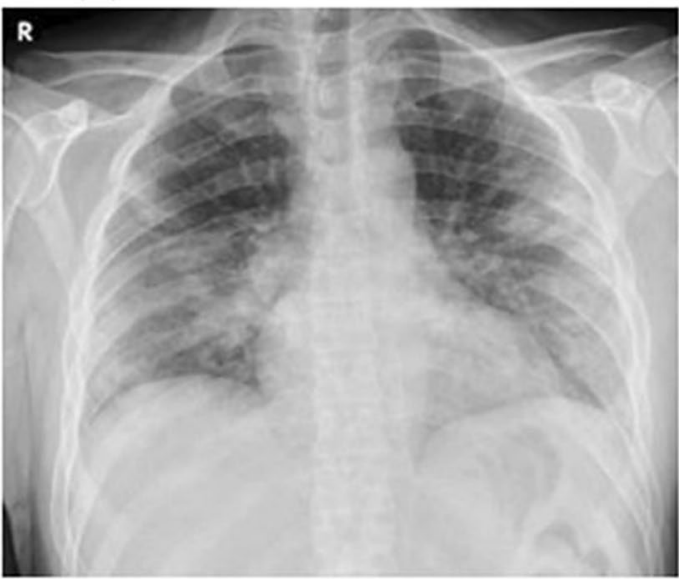

(d) COVID-19 Pneumonia

Fig. 5 Example of Chest X-Ray Images a Normal Image b Bacterial Pneumonia Image c Viral Pneumonia Image d COVID-19 Infected Image [87] 


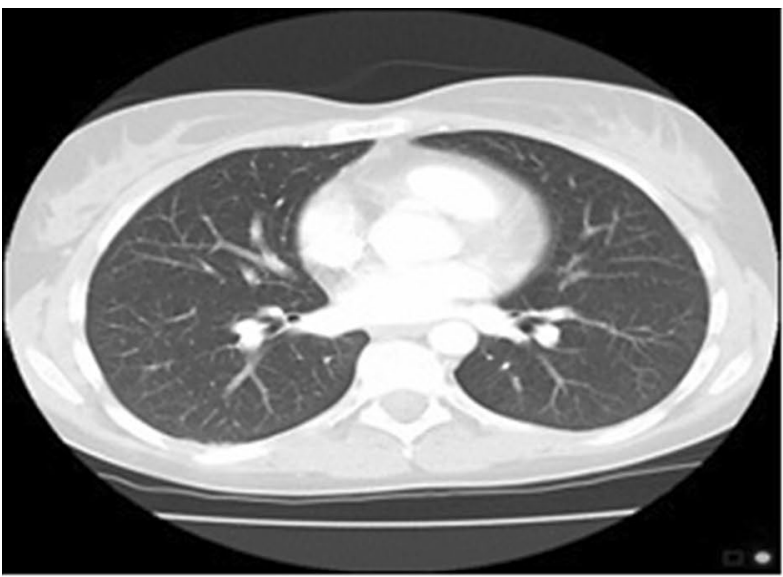

(a) Normal CT Scan

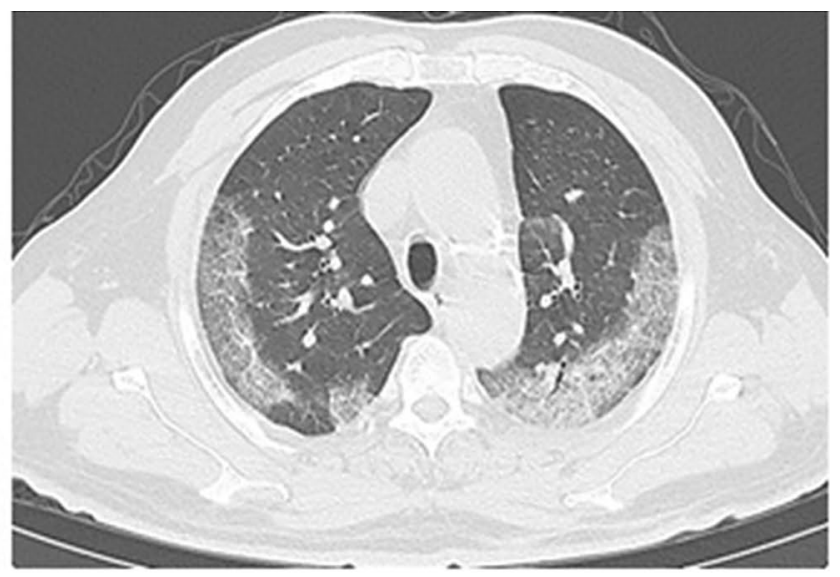

(b) COVD-19 CT Scan

Fig. 6 Example of CT Scan Images a Normal Image b COVID-19 patient's Image [88]

The model study of this epidemic statistics has fascinated the establishments concerning the detection and prediction of COVID-19. This is moreover eminent truth that the nation's governments with other authorities of different countries have always relied on several types of illness prediction schemes that conduct the accomplishment of inventive generated strategies and determining the effectiveness of previous decisions. In the recent time of the health crisis, the worldwide legal authorities are increasingly emphasizing the use of various speculative representations with COVID19 statistics to construct the informed decisions. This will facilitate them towards the execution of suitable controlling measurements [95] and also improve outburst COVID-19 suppression, recognition, and forecasting agreements.

More global COVID-19 predicted calculations are accessible at $[1,96,97]$. As the survey includes numerous challenges to resolving the COVID-19 and its related issues, there is a great need to reinforce the skills required by traditional methods, especially to strengthen the resilience of predicted outcomes.

Digital technology had a high impact on resolving critical health care and the associated curative targets. Most of the innovative technologies use the big data-analytics model, 5G IoT (Internet of Things), AI (Artificial Intelligence) with ML models, blockchain technology [98]. It can be a wellingrained fact that DL has firmly expanded considerably within the sector of ML through its use in every aspect of individual health [99]. For example, the study of [95] has been gathering coronavirus epidemic data in Italy. The work accomplishment in ML with other Soft computing methods forecasts the likelihood of an explosion that allows executives to better plan for controlling the disease and associated economic systems. In the DL approach, data size and its quality perform a significant part in producing very effective results. The COVID-19 began in Wuhan, China as well as the people who are prone to be affected by this epidemic, were acknowledged towards dedicated hospitals in Wuhan to perform illness control policy [100]. The details of the patient's data with confirmed COVID-19 positive case were affectively collected and analyzed by the researchers to help in ML with DL research behaviors [101]. In different countries as China, South Korea, Iran, and Italy, the trending data is used to gather COVID-19 related information [102]. Another source of illness prediction data includes the curvature curves and appropriate capacities from affected people in Hubei in China, with a visible arc [103] in envisioning the locations for potential outbursts.

Liu et al. [104] used ML mechanisms to predicting the COVID-19 conditions in different areas of Chinese states by forming the clusters which permit the synchronous development of geospatial areas. The DL scheme in medical image practices assists in performing methods for epidemic modeling to translate the numerous COVID-19 positive compared with the number of recovery cases found globally. COVID19 cases ranging from no symptoms to severe respiratory suffering and manifold respiratory damaged elements. The coronavirus positive individuals examined at Anhui Medical University Hospital, China that discloses the people having SARS-CoV-2 detected at starting through RT-PCR. The experimental results have examined the gene sequence structure that compared with epidemic positive SARS-CoV-2 reports. Wuhan central hospital analyzed several patients having frequent pathogen detection utilizing Chest CT scan images [105].

The chest CT scan of the lungs distinguished the presence of COVID-19 disease [106], moreover declared to detect the COVID-19 more rapidly than the traditional methods as RT-PCR. It gives importance to the study of COVID-19 in 
which same symptoms with additional categories of pneumonia, that is quite difficult to correct classification. To overcome this complexity, the main target study includes traditional DL methods, using key characteristics integrating with medical image processing applications for accurate identification of COVID-19 positive CT scans, and minimizing the calculating errors to recover the efficiency of estimation results.

The CNN model with entirely integrated, used for COVID-19 diagnosis is shown in Fig. 7. The preference is mainly due to the strongly active impact of the COVID19 pandemic and the unpredictability in its proceedings nationwide.

\subsubsection{The Architecture of CNN Transfer Learning}

A deep neural network is under the branch of ML, which is encouraged by brain formation. This technique is widely used in recent years persist to prove remarkable performance in the area of medical imaging domain. Using DL techniques, attempts are made to draw visualize meaningful results from the medical image data. CNN models have been applied effectively in various fields such as lesion segmentation, classification by medical imaging tasks such as CT Scans, MRI images, X-rays. As an outcome of data analysis, the detection of ailments such as brain tumors, breast cancer, diabetic retinopathy, and skin cancer is easily mentioned in [107-111].

A CNN is a category of deep networks which is widely used in image diagnosing problems [112]. When it comes to CNN performance, the computerized medical images as input and converted to the dedicated resized format. The training procedure, evaluate the consequences of distinctions on the label and predict new images with them. CNN has three layers with a convolutional layer, pooling layer, and fully connected layer to execute various functions efficiently. The feature selection procedure is performed on the convolutional and pooling layers. Conversely, the classification task is executed on the fully connected layer. A detailed explanation of the layers is mentioned below:

Convolutional Layer The convolutional layer is the root layer of deep network systems. It is highly reliable for uncovering the features of the data sample. In this layer, the input image is transferred to the filter. As an outcome, the values are generated in the form of a feature map. Different kernels are applied in this layer so that low and high-level features are extracted from the medical images [113]. A kernel is a matrix having $3 \times 3$ or $5 \times 5$ shape dimensions for conversion with an input matrix. The parameter called "Stride" is defined as the number of steps adjusted to switch over the input matrix. The result of a convolutional layer is given as:

$x_{k}^{L}=f\left(\sum_{i=1}^{n} m_{k}^{L-1} * p_{i}^{L-1}+q_{k}^{L}\right)$

where $x_{k}^{L}$ is the kth feature map in the layer $\mathrm{L}, m_{k}^{L-1}$ represents kth kernels in the layer of $\mathrm{L}-1, p_{i}^{L-1}$ determines the ith feature map in the layer of $\mathrm{L}-1, q_{k}^{L}$ represents the bias of the kth feature map in the layer of $\mathrm{L}, \mathrm{n}$ is the total number of features in layer L-1, and “*” represents the vector convolution method.

Pooling Layer The pooling layer is the second layer in the deep neural networks. This layer is often performed on the feature map which is designed to lessen the number of features and network constraints using parallel statistics. In this layer, the global average pooling and max-pooling are used. In the max-pooling, it chooses simply the maximum value through the matrix size defined in each of the feature

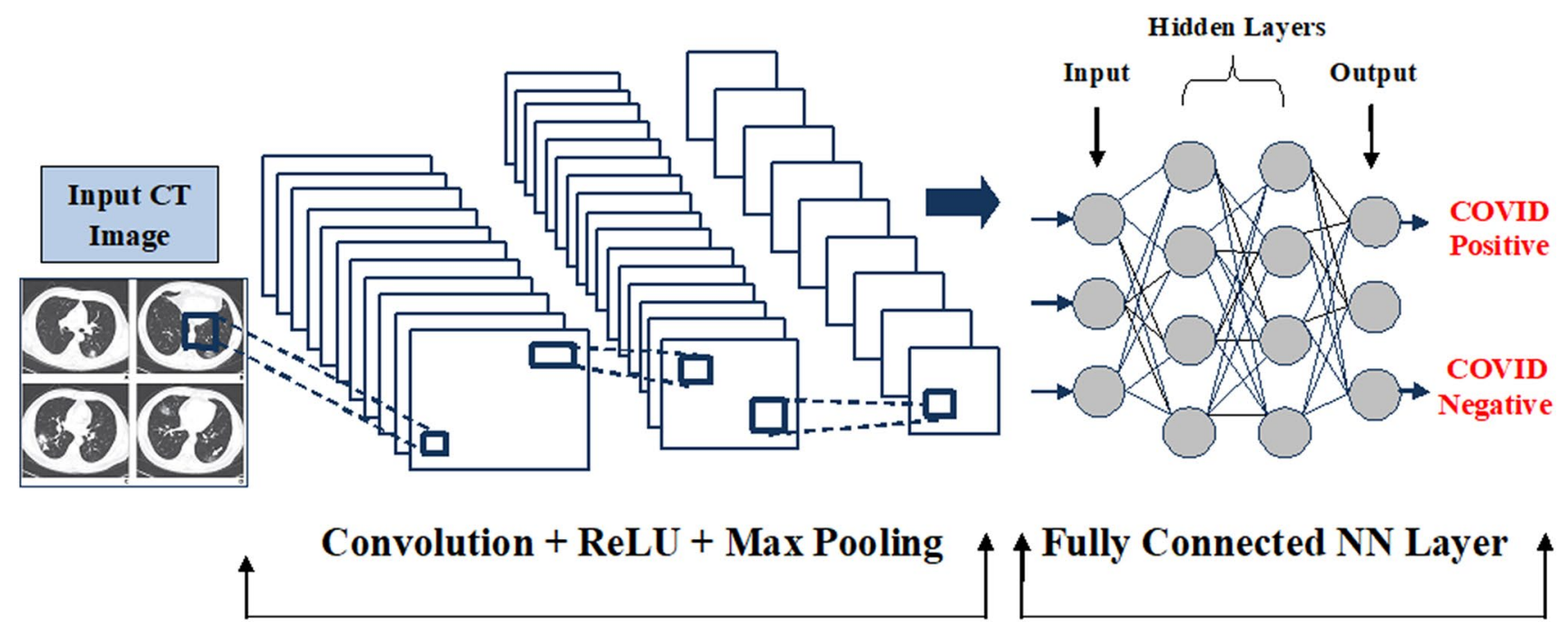

Fig. 7 Example of a fully connected CNN for detection of COVID-19 in CT scan medical images 
maps. It will lead to a reduction in the release of output neurons. The global average layer is simply performed earlier than the fully connected layer, which reduced the data to a single scale [114].

Fully Connected Layer The last and most significant layer of CNN is the fully connected layer. It performs as a multilayer perceptron in the deep neural network. The ReLU (Rectified Linear Unit) activation function is usually used by a fully connected layer. The Softmax function is applied for predicting the multiple cases from the medical images in its last layer. The statistical calculations of both activation functions are described below:

$\operatorname{ReLU}(y)= \begin{cases}0, & y<0 \\ y, & y \geq 0\end{cases}$

$\operatorname{Softmax}\left(\mathrm{y}_{\mathrm{i}}\right)=\frac{\mathrm{e}^{\mathrm{y}^{\mathrm{i}}}}{\sum_{\mathrm{x}=1}^{\mathrm{n}} \mathrm{e}_{\mathrm{x}}^{\mathrm{y}}}$

where $y_{i}$ and $n$ present the input data and the number of classes defined respectively. The neurons in this layer have full connectivity with every activation function in the earlier layer.

CNN Pre-trained models In medical data analysis, there is a problem faced by investigators related to the restricted amount of available real datasets. DL models usually require a large number of data. Categorization of this data professionally is a very expensive and time-consuming process. The great advantage of the transfer learning approach is that it permits a few training datasets and needs low computation costs. The information obtained from the CNN pre-trained model is passed to the training phase on a large dataset.

In Fig. 8, there is an example of deep CNN based on pretrained models such as ResNet50, ResNet101, ResNet152, InceptionV3, and Inception-ResNetV2 for the multi-classification of COVID-19 chest CT scan images. There are three binary classes such as Binary class 1 represents the classification between COVID-19 and normal images, Binary class 2 determines the classification between COVID-19 and viral pneumonia, and Binary class 3 indicates the distinction between COVID-19 and bacterial pneumonia. Moreover, transfer learning is obtained by utilizing ImageNet data to conquer the inadequate data and computational training time.

\section{ResNet50}

The ResNet (Residual Neural Network) model is an enhanced form of the deep neural network. It includes some alternatives among the layers to solve the defined problem. As an outcome, it avoids distortions that take place as the network becomes deeper and more complicated. Moreover, the restricted blocks are extensively used to speed up the training in the ResNet model [115].
The ResNet50 is a pre-trained model with 50-layers on the ImageNet database. This database contains above 14 million images, where more than 20,000 classes are designed for image identification purposes [116].

2. ResNet101 and ResNet152

The models, ResNet101 and ResNet152 contain 101 and 152 layers correspondingly because of stacked residual blocks. In this study, it is possible to load a variety of pre-trained networks from the ImageNet database trained in over a million images [116]. As the result, the dedicated network has performed feature extraction of various images. The model has an input image dimension of $224 \times 224$.

3. InceptionV3

The InceptionV3 is a CNN pre-trained model from the Inception group. It is designed to make various enhancements such as categorize smoothening, factorized $7 \times 7$ convolutional, and employing the secondary classifier. It is used to circulate the class information all over the network with the help of batch normalization [117].

4. Inception-ResNetV2

The CNN model, Inception-ResNetv2 has trained over a million images from the ImageNet database. The model has 164 deep layers and can categorize the images into thousands of object classes such as COVID-19, bacterial pneumonia, viral pneumonia, or normal. As an outcome, the model has been trained to extract meaningful features from a large set of images. The network with an input size of $299 \times 299$, and the corresponding output is a set of approximated case probabilities [118].

Table 3 determines the summary of COVID-19 benchmark imaging datasets with their corresponding format. In the study, it accumulated a list of open-access datasets, which is widely used for predicting the multiclass entities as COVID-19, bacterial and viral pneumonia, and normal cases. The researchers utilizing and compiling the lung CT scan slices and chest X-ray images which are obtained from the various publicly accessible data repositories.

\subsection{Coronavirus Transmitted Indication}

In December 2019, while the community was meant to be waiting for the celebration of the new year 2020, a few pneumonia cases originated from the COVID-19 epidemic [129] were generated report in the Wuhan city, China. The researchers in [130] have mentioned that a huge scale of individuals is affected positively due to wet mammals in the city of Wuhan, measured in the form of zoonotic as a source of COVID-19. Ultimately, many cases widely spread throughout the city of China, and worldwide gave it a rank of an outbreak [131]. 


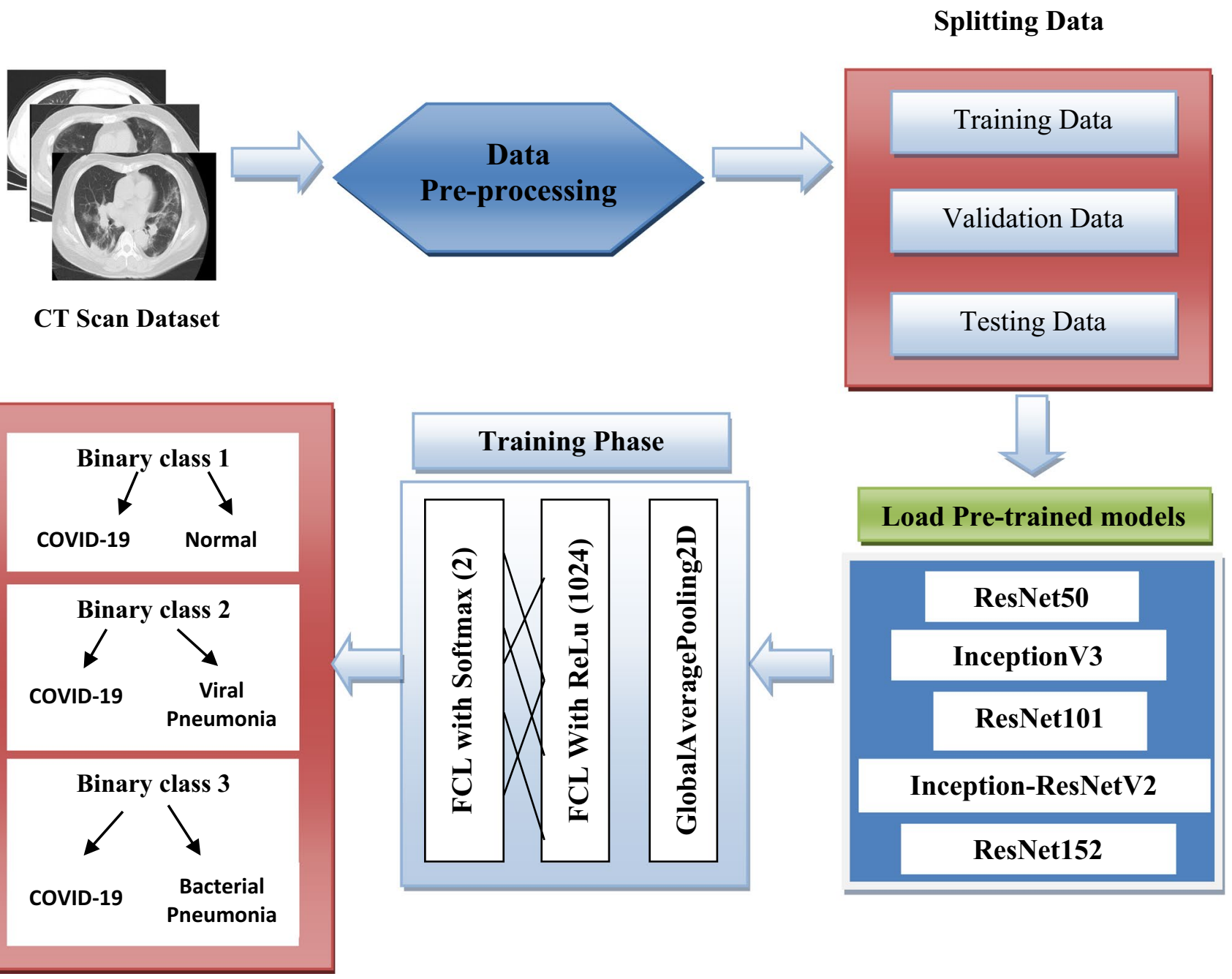

Fig. 8 Diagrammatic representation of CNN pre-trained models for diagnosis of COVID-19, normal, bacterial, and viral pneumonia

Lu et al. [132] projected two kinds of snake classes as probable basins of the coronavirus pandemic. Xu et al. [133] established the pangolins as a transitional center for the unexpected COVID-19. The study in [134] has shown that the investigation of the genomic sequence of COVID-19 was so comparable with bat-originated SARS (Severe acute respiratory syndromes). In the same way, Malik et al. [135] proposed various animals like bats, pangolins, civet cats, etc. considered to be a possible cause of the SARS-Cov-2 virus in humans.

There are various applications have been developed by focusing on the medical image processing, computer vision, ML, AI, which assists in monitoring, testing, detecting and controlling the main impact of pandemic worldwide. For example, Defensive types of equipment, ventilators, automated sanitization, ventilators, breathing apparatus used to treat affected people and protect healthcare experts ensure the presence of virus infection as well. Temperature testing is widely used to assess human temperature as high temperature recognizes the key symptom. The social isolation scheme is performed for perceiving the safest less proximity from the COVID-19 positive patients and visual-conducted robotics mechanisms have been followed in this concern. The government authorities in Australia have been commenced a 'Dragonfly' as a UAV (Unregulated Airline Vehicle), to quickly deploy drones for detecting the COVID-10 illness in distant locations accurately among citizens.

The partnership with the University of Waterloo as DarwinAI organization has launched Deep-CNN as "COVID-Net" for diagnosing the coronavirus [136] in chest radiographic images. Medical image practices with computer-vision methodologies are widely used in the mass invention of healthcare pieces of equipment being used safely by hospital faculties. The resources help to prevent the spread of the virus by reducing the human distance. It proved their specialty in detecting and diminishing the air-borne 
Table 3 An outline of COVID-19 open-access databases

\begin{tabular}{|c|c|c|c|c|c|}
\hline Ref & Database Name & Number of patients & Data format & Country & Dataset link \\
\hline [119] & $\begin{array}{l}\text { SIRM (Italian Society of Medical and } \\
\text { Interventional Radiology database) }\end{array}$ & 68 patients & JPEG & Italy & $\begin{array}{l}\text { https://sirm.org/category/senza-categ } \\
\text { oria/covid-19/ }\end{array}$ \\
\hline [120] & Radiopaedia database & 101 cases & JPEG & Worldwide & $\begin{array}{l}\text { https://radiopaedia.org/articles/covid- } \\
19-4 ? \text { lang=us }\end{array}$ \\
\hline [121] & MosMed: Chest CT scan database & 1110 cases & NIfTI & Russia & $\begin{array}{l}\text { https://mosmed.ai/datasets/covid19_ } \\
1110 /\end{array}$ \\
\hline [122] & $\begin{array}{l}\text { UCSD (University of California San } \\
\text { Diego) Chest CT database }\end{array}$ & 349 CT scans from 216 cases & PNG & Worldwide & $\begin{array}{l}\text { https://github.com/UCSD-AI4H/ } \\
\text { COVID-CT }\end{array}$ \\
\hline [123] & COVID-19 ML database & $930 \mathrm{CT}$ scans from 461 cases & JPEG, NIfTI & Worldwide & $\begin{array}{l}\text { https://github.com/ieee8023/covid- } \\
\text { chestxray-dataset }\end{array}$ \\
\hline [124] & $\begin{array}{l}\text { COVID-19 CT segmentation data- } \\
\text { base } 1\end{array}$ & 100 slices from 50 patients & NIfTI & Italy & $\begin{array}{l}\text { http://medicalsegmentation.com/covid } \\
19 /\end{array}$ \\
\hline [125] & CT Scan segmentation database 2 & 800 Lung slices from 9 cases & NIfTI & Worldwide & $\begin{array}{l}\text { http://medicalsegmentation.com/covid } \\
19 /\end{array}$ \\
\hline [126] & $\begin{array}{l}\text { COVID-19 CT Lung slices and infec- } \\
\text { tion segmentation }\end{array}$ & 20 patients & NIfTI & Worldwide & $\begin{array}{l}\text { https://zenodo.org/record/3757476\#. } \\
\text { YSDS6Y4zbIV }\end{array}$ \\
\hline [127] & AIforCOVID medical image database & 983 cases & DICOM & Italy & https://aiforcovid.radiomica.it/ \\
\hline [128] & Eurorad dataset & 50 cases & JPEG & Worldwide & $\begin{array}{l}\text { https://www.eurorad.org/advanced- } \\
\text { search?search=COVID }\end{array}$ \\
\hline
\end{tabular}

JPEG joint photographic experts group, $P N G$ portable network graphics, NIfTI neuroimaging informatics technology initiatives, DICOM digital imaging and communications in medicine

elements, which have great potential to infect a large number of people with the COVID-19 illness. The disease indication system which considers the data science tasks emphasize responding to various conditional questions or challenges associated with the pandemic [137]

The biggest dispute of the occurrence condition is modeling the approach where collecting of datasets, data analysis, and live dataset reporting accordingly. Department of Health-care, New Zealand generated a report [138] that planned a comprehensive data analysis that amplified the general population, time, and various epidemiological parameters. This study understands the collision and transmission factor of the COVID-19 pandemic depending on different parameters like age, region area, gender, past travel records, and daily live records from any such observational samples by using a deep network with ML mechanisms.

Pourghasemi et al., Saha et al. [139, 140] performed generate a CSSE Tool [141] named GIS (Geographic Information System) for locating the affected people with this illness. The novelists [142] have compiled a GIS tool system with ML and SVM (Support Virtual Machine) for measuring the risk factor of coronavirus in the states of Iran as Fars. In terms of information, the internet serves as a great source to achieve data samples on this COVID-19 illness. In addition to this pandemic related data, the number of COVID-19 positive cases, mortality rate, and the recovery rate are also offered to access in the dashboard of Johns Hopkins, University of Medicines [141]
Afterward, WHO also introduced the dashboard of COVID-19 [97], which works on Arc-GIS. The mobile application as Aarogya Setu [143] offers constructive detailed information regarding COVID-19 positive cases in the provincial areas of India. The medical image processing techniques with DL applications have also been used for the development and authenticate the model representation [144] at Renmin University of China. It is usually gathered 46,096 anonymous images of different 106 patients in hospitals. The admitted patients lied into two classes; firstly, COVID-19 positive patients and secondly, patients with some other common disease.

51 positive cases are diagnosed with coronavirus disease. The medical team examined and analyzed the 21 Chest CT scan images with COVID-19 positive performed at [144] Renmin University of China. The system emphasized on DL is developed to make certain conclusions for physicians to early diagnose the contaminated pneumonia COVID-19 cases to manageable controlling of the pandemic.

\subsection{Detection and Therapeutic Aspects of COVID-19}

The pandemic, COVID-19 is termed as a cluster of numerous viruses. When the patients have been infected with the defined ailments, then the symptoms may resemble acute respiratory disease or an infectious common cold. For example, MARS-CoV (Middle East Respiratory Syndrome), SARS-CoV (Severe Acute Respiratory Syndrome) is one of the most serious ailments [145] with the same properties as 
COVID-19. Globally, many people get affected with outburst COVID-19, that's why countries have decided to announce a nationalized closure with millions of citizens firmly being quarantined [146-149]. In this kind of critical situation, outbursts predictions, and virus-transmission indicator tools which used DL and machine imaging techniques are highly potent for diagnosis and therapeutically active for coronavirus. Also, it helps to support physicians with the starting diagnosis process and quick detection of the virus accurately and efficiently.

Biomedical imaging techniques like CT Scans images are highly supportive in diagnosing disease quickly and accurately and are no different from the coronavirus. The COVID-19 positive CT scan images are considered to have better sensitivity $80-90 \%$ than RT-PCR and specificity 60-70\% lower elevation [150, 151].

The DL with the medical image practices engages in recreation with a significant aspect of isolating the COVID-19 positive patients and non-infectious people. The symptoms of COVID-19 are closely related to common pneumonia. The Spanish Hospitals have been viewed the mechanisms of collection of features in the criteria of the detection process. Though, several sources have been detected X-rays as a substitute investigation [152].

Another unexpectedly work in $[153,154]$ has proposed a differentiation among the ultra-sonography and CT scans where observed that ultra-sonography having more reliable results against detecting the COVID-19 as compared to chest X-rays. Qin et al., Zou and Zhu [155, 156] have highlighted the need for a large-scale dataset related to COVID-19 for image detection employing PET-CT. Currently, the CT Scans with AI techniques assisted in the diagnosis of the COVID-19 positives with dissimilar symptoms. Jin et al. [157] planned to generate situational reports on various tools to sustain the detection process of COVID-19. The report contains some guidelines related to population avoidance, epidemiological features, detection of COVID-19 disease.

Standford University offered some useful details, tools, investigational studies, models, and financial support for the coronavirus study. A collaborative research endeavor with dedicated COVID-19 databases facilitates the creation of an inclusive analysis of medical imaging with DL methods for acquisition, illness diagnosis, cure management, as well as vaccine progression. Various medical imaging databases are considered as-(1) IEEE 8023 Chest X-rays and CT scans dataset on the COVID-19. (2) Mathematical health center in medical image processing with AI mechanisms for coronavirus detection. (3) CNN with AI approach, University of Waterloo. (4) The Consortium as RadiologyAi with coronavirus positive $\mathrm{CT}$ scans.

The DL-related experimental models have proven to be reliable and efficient results by using standard image datasets by combining the various medical image processing methods. This approach analyzed that the parameters as sensitivity, specificity, and precision for detecting the outbreak through RT-PCR, gives low efficiency as compared to radiographic patterns with CT scan images at an early stage of illness.

Various types of CNN techniques encompass to study by $\mathrm{Xu}$ et al. [158] detecting the CT scan samples from three different kinds of classes as (1) COVID-19 (2) Pneumonia Infection (3) Normal (No infectious disease). They also worked on traditional DL models with 2D and 3D methods that included some comprehensive studies on the foundation of thoracic CT scans, achieving sensitivity, specificity, AUC, and accuracy for infectious Vs. non-infectious test reports.

\subsection{Vaccine Availability and Remedy Research}

The R\&D Department, WHO has begun endorsing the progression of detection, vaccination, and treatments [159] for this unexpected COVID-19. This rapidly spreading virus requires immediate diagnosis to boost the possibilities of high recovery rate in affected people and offering great chances for immediate treatment. The detection method is therefore an important part to comprehend the number of COVID-19 positive cases and distinguish people who are defiant and probably confined from the infectious disease. It is mandatory to establish a stable and effective fresh vaccine adjacent to the highly dangerous coronavirus. DL and medical imaging have a high probability that assists in the identification of the vaccines and related remedies to cure COVID-19 at an early stage.

ML techniques collaborated with DL methods for accurate training with large data samples of dedicated medical compounds. Some of the composites can boost the human's immune system, while others do not have. In this criterion, DL and ML techniques can make composite patterns that assist in building high immunity against viruses in a short period. The novelists can further use the ML methods to identify whether that novel discovered chemical composites in the vaccine can be used in the future or not. In this kind of approach, ML and DL methods are most widely used in the practices of determining the vaccines/cure remedies [160].

Disease vaccines form closely the same or element of the antigens which originated the virus. When the vaccines have been commenced into the human body, it will work the immune system to make certain antibodies to detect and counterbalance the virus infection. The virus infection often multiplies rapidly as well as their antigens are more susceptible to the mutation that avoids the detection of immunizer. Multiple attempts to produce the vaccine which differentiates the T-cell antigenic of SARS-COV-2 illness are mentioned by Qiao et al. [161]

Tayebi et al. [162] followed CNN architecture as a DL method for predicting the CIR (Cross Immuno-Reactivity) 
in antigenic vaccination. Vaishya et al. [163] developed AI-based techniques with DL that will be used to provide hospitality services. kinds of tools emphasizing medical imaging such as CT scans or X-rays to scan the mild-stage cases diagnose new infections and supervise the advanced level diseases to identify COVID-19 ailments. The applications of medical imaging have worked with DL that assists to identify different techniques to discover remedy-making mechanisms to battle with COVID-19. This emergence can set the final goal stage or an active anti-viral infection.

Zhang et al. [164] proposed to utilize (DFCNN) DeepFeatured CNN to recognize the latent vaccine for coronavirus. Beck et al. [165] suggest the DL model with targeting drug use against COVID-19 in the Prediction of antibacterial drugs for profit. Ong et al. [166] performed a transformable vaccine with ML techniques for mounting the development of a vaccine against the virus is introduced. The virus and bacterial infection have been tested to improve the treatment of SARS-CoV-2 and MERS-Cov-2 ailments. In the further steps, the vaccine transpose tool as Vaxign and ML based tool as Vaxign-ML used to forecast the applicants for coronavirus vaccines.

Currently, there are approximately more than 50 coronavirus vaccine applicants registered in the trials. WHO [167] mentioned that teamwork with businesses, International healthcare organizations, scientists in the course of Accelerator (ACT) to make a speedy response against COVID19 . With the availability of a secure and effective vaccine, COVAX (escorted by WHO, CEPI \& GAVI) will help to achieve reasonable admittance and allocation of the vaccines in the countryside. The high-risk affected people will be at high precedence in vaccine trials.

\subsection{The Drawback of DL-Based Medical Imaging on COVID-19 Outbreak}

Even though millions of infected people with COVID-19 have been discovered, and there is still a lack of a large public database that targets especially the absent infection tests. The correctness of DL representation is fully dependent upon the accessibility of data that leads to being a major problem in the COVID-19 scenario. The effectiveness of the DL model is doubtful because of inadequate data. Also, the convenience to access the $\mathrm{CT}$ scans or X-rays from large demographics leads to complicate to assemble in the healthcare departments.

Moreover, the performance of the DL-based policymaking approach requires entry to ethical indicators such as employment, education, personal history. These types of data samples are much needed to implement how individuals stay healthy as possible even later being infected with the COVID-19 virus.
The discussed limitations are crucial to achieving advanced systematic assessments. However, as mentioned in the paper, several studies have been accomplished consistently to eradicate such confronts and to gain further transparency in the diagnosis of COVID-19 [168].

\subsection{Outline of the Section}

After the critical overview of the developing literature, clear that DL and medical imaging tasks perform a significant part in fighting against the pandemic COVID-19 throughout a variety of potential applications considering outburst prediction, the coronavirus transmitted indications, detection and therapeutic aspects of the disease, and vaccine discovery and remedy research. The extensive research and selected indicators are summarized in Table 4.

\section{Lessons, Discussions, and Future Directions}

Even though DL has expanded a lot of impetus, attractiveness and produced good results with normal two- dimensional images, there are clear boundaries to achieve the same level of implementation in the use of medical image methods. The reputed research study in this concern is ongoing with some parts of the lessons have mentioned below:

- There is one of the biggest barriers to the unavailability of large real databases with highly premium training images. To overcome this issue, data augmentation is a potential approach.

- CT Scans \& MRI images are used typically based on 3D with applied some CNN pre-trained models as ResNet-50, ResNet-101, MobileNet SqueezeNet, InceptionV3, Xception, NasNetLarge, NasNetMobile, DenseNet121, DenseNet169, DenseNet201, InceptionResNetV2, etc. to achieve better results.

- The large dataset is split into training, validation, and testing set with a ratio of 70:20:10 to optimize the model's performance. $10 \%$ of the data sample can be considered as test data for testing the performance of the model.

- The unusual process for accumulate image datasets has become one of the foremost questions in medical image processing. The best way to solve this problem is to use the mechanisms of transfer learning approach that will make the pre-processing task more accurate and efficient as well as eliminate the acquisition and scanning issues.

- It is anticipated that cloud-oriented platforms will compete for an important role in the future expansion of computational deep network applications. Using cloud computing provides a key to handling large data samples. It also assists to increase intelligence and diminish 


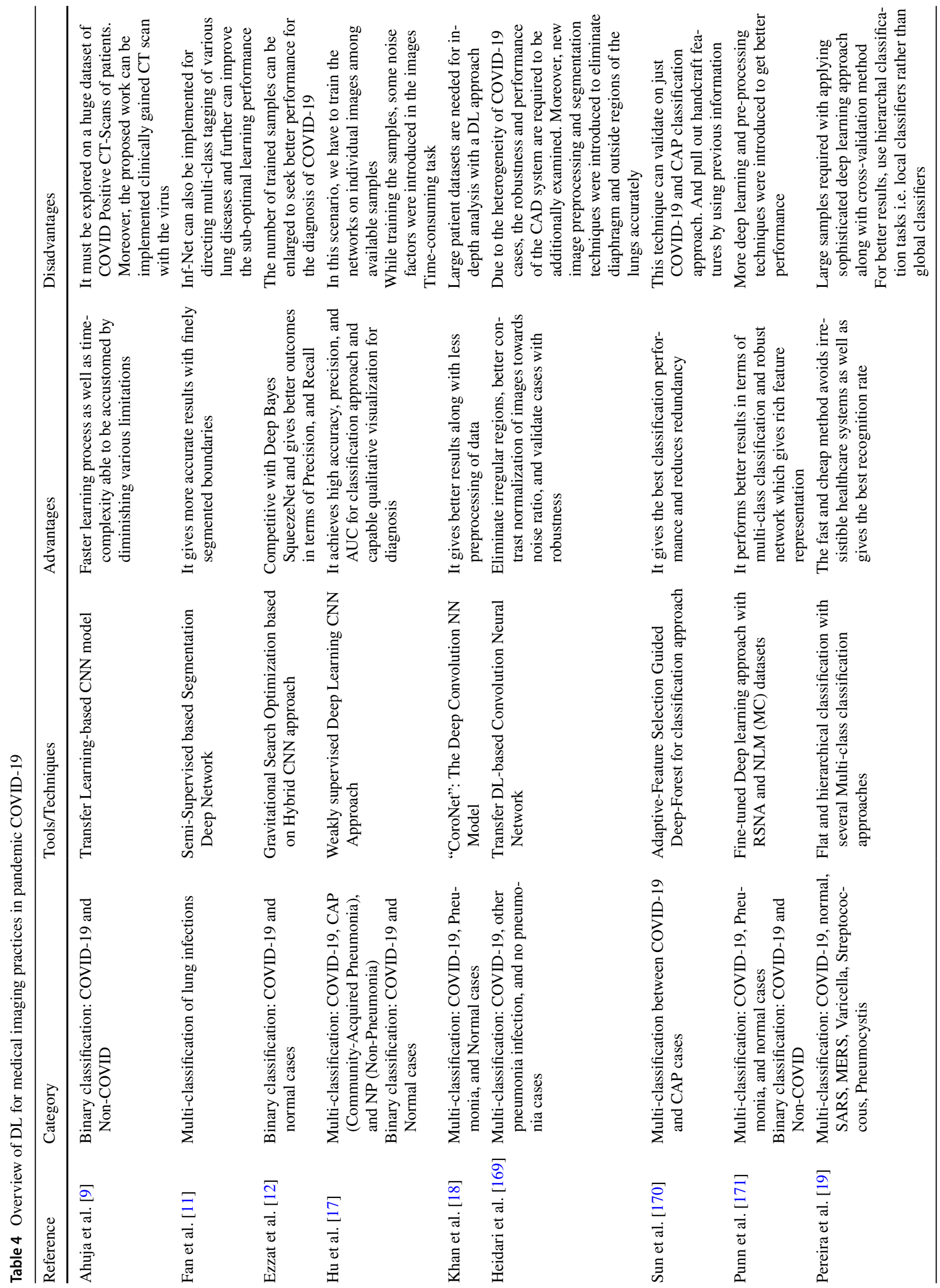


costs. In addition, it provides the flexibility to train the DL frameworks.

\subsection{Discussion}

In Table 5, different techniques are compared with some state-of-the-art methods for diagnosis the COVID-19 infection and yield several new observations as mentioned below:

\subsection{Challenges and Issues}

The confrontations and issues related to the implementation of DL in medical image processing to control the epidemic COVID-19 in the smart urban colonies are listed below:

Confidentiality - Access to high-quality images with large data samples becomes a major confront while considering the confidentiality of coronavirus-affected individual's details.

Diversity outburst pattern-Data emergence followed the major aggressive variations of COVID-19 infection in different countries which lead to less reliability for predicting it.

Directives and precision-The global countries have strictly followed rules for compliance with the allocation of the COVID-19 dataset. Although, the datasets and case studies should be firmly collected from COVID-19 patients in a short time frame. So, this whole criterion seems very tough to handle out.

Inconsistency of screening actions in different hospitals plays a major problem associated with (FN) False Negatives in data label classes.
The indication of both pneumonia and coronavirus are quite similar. Identifying the best DL method for diagnosis COVID-19 with high efficiency still leftovers as a significant challenge.

\subsection{Future Directions}

Besides, the COVID-19 infection gets fully tracked based on the data which is collected from the numbers of infected patients worldwide.

1. The inherited chain performs to a great extent because the COVID-19 virus has a high rate of mutation. The outcome of the false diagnostic test is distressing and there are high chances of FN for an infected patient genuinely suffering from the illness. The inspection on the COVID-19 diagnosis testing followed by the committee determines the faster spreading rate from individual to individual [175].

2. In the COVID-19 situation, the future indicators related to some limitations of the large dataset with extra clinical information assist in improvement for disease prediction. Moreover, accessible datasets are small in size which is insufficient for correct predictions. The future advice is to use transfer learning which detects irregularities in smaller size datasets and produces efficient results. In the early diagnosis of COVID-19, algorithms are used to speed up the predicting process within less time and with minimal resources.

3. The transfer learning is used for the detection of COVID-19, where the time and accessibility of training data being as a crucial part. Also, this approach is

Table 5 Comparison with the other state-of-the-art methods for COVID-19 binary and multi-classification

\begin{tabular}{|c|c|c|c|c|c|c|}
\hline Authors & $\begin{array}{l}\text { Number of cases (COVID- } \\
19)\end{array}$ & Data Format & Methodology & $\begin{array}{l}\text { Two-class } \\
\text { accuracy } \\
(\%)\end{array}$ & $\begin{array}{l}\text { Three-class } \\
\text { accuracy } \\
(\%)\end{array}$ & Sensitivity (\%) \\
\hline Narin et al. [20] & $\begin{array}{l}\text { Total cases }=100 \\
\text { COVID-19 }=50\end{array}$ & X-ray & ResNet50 & 98 & - & 96 \\
\hline Khan et al. [18] & $\begin{array}{l}\text { Total cases }=221 \\
\text { COVID-19 }=29\end{array}$ & X-ray & Xception & 98.8 & 94.52 & 95 \\
\hline Wang et al. [13] & $\begin{array}{l}\text { Total cases }=237 \\
\text { COVID-19 }=119\end{array}$ & CT scan & Modified Inception & 82.9 & - & 81 \\
\hline Wang et al. [172] & $\begin{array}{l}\text { Total cases }=300 \\
\text { COVID-19 }=100\end{array}$ & X-ray & COVID-Net & 96.6 & 93.3 & 91 \\
\hline Song et al. [173] & $\begin{array}{l}\text { Total cases }=57 \\
\text { COVID-19 }=30\end{array}$ & CT scan & ResNet50 & 86 & - & 79 \\
\hline Ozturk et al. [8] & $\begin{array}{l}\text { Total cases }=1127 \\
\text { COVID-19 }=127\end{array}$ & X-ray & DarkCOVIDNet & 98.08 & 87.02 & 90.6 \\
\hline $\begin{array}{l}\text { Rahimzadeh and Attar } \\
\text { [174] }\end{array}$ & $\begin{array}{l}\text { Total cases }=11,302 \\
\text { COVID-19 }=31\end{array}$ & X-ray & ResNet50V2 + Xception & 99.5 & 91.4 & 80.53 \\
\hline Apostolopoulos et al. [10] & $\begin{array}{l}\text { Total case }=1427 \\
\text { COVID-19 }=224\end{array}$ & $\mathrm{X}$-ray & MobileNetV2 & 96.7 & 93.5 & 98.6 \\
\hline
\end{tabular}


usually performed on pre-trained models in academia, open-source society, research organizations, etc. to save resources and time as well. DL produces good outcomes when a large set of data samples are obtained, but with the transfer learning mechanism, a similar outcome can be attained with an inadequate dataset size [176].

4. In the outbreak condition, the accessibility of predefined data is an ultimate challenge. That's why transfer learning has great potential to serve the aim of the true discovery of COVID-19. The researchers [177] can be focused on the lung CT scans, and X-ray images of infected patients collected from an open-public repository like GitHub. These medical images were identified to choose the suitable sample among the total images.

5. Other bacterial infections such as pneumonia, viral pneumonia, and normal case dataset were collected from the open repository named Kaggle. The pre-trained CNN models incorporated ML techniques for the multi-classification of COVID-19 disease into different classes.

6. The methodology was discussed in [10] as the database of 1427 images containing 224 cases of COVID-19, 700 Common bacterial pneumonia, and 504 normal images. Therefore, the generation of COVID-19 data repositories is a critical requirement. It leads to developing the prognosis, diagnosis, and treatment therapies to fight against the disease. The future epidemics in health smart cities will be predicted shortly.

As discussed in traditional studies, there are various DL applications performed by using CNN pre-trained experimental models where the radiological images are very common. However, the use of these technologies in real-time practice becomes a major challenge. The background outlines should facilitate the computer-assisted systems that monitor the patient reports, data pre-processing mechanisms, and testing process for different AI techniques to ensure data intelligibility.

The citizens of today's world are more fortunate than any old generation, who has seen the Spanish flu outburst in 1918 as a new generation has given superior technology. AI mechanism is widely used in the subject of human existence. For example, AI is used in the future for the development of self-directed robots and equipment for antimicrobial treatment, health care in hospitals, delivery of medicines along with some essentials to patients. AI is meant to be collaborated with NLP (Natural Language Processing) methodology to develop a discussion box. It is widely used for remote communication with COVID-19 infected patients and offers conferences throughout the time of emergency.

In addition to the benefits summarized above, the use of AI can cooperate in eliminating the falsehoods distributed in social media. The implementation of AI focuses on authority policies, epidemic prevention policies, and the rapidly spreading viruses and infections. It ensures realistic information by eradicating all the preventable panic patterns. In this time of crisis, the major concern is the expansion of the original vaccine against the COVID-19. AI has great potential in this study of genetic and protein synthesis of the coronavirus to speed up drug detection. AI with the DL approach will be able to detect the suitable antibiotic from 100 million large molecular datasets. It is an interesting indication of future research advice to defeat the COVID-19 epidemic.

\section{Conclusion}

Along with solutions to fight the epidemic COVID-19, DL is considered a high-quality strategy to offer smart intellectual resolutions. Inspired by numerous DL programs for medical imaging techniques over the past decade, it is necessary to overview the recent novel COVID-19 for healthy smart communities. DL has been carried out a variety of solutions such as prediction of coronavirus disease; virus transmitted diagnosis, detection, and therapeutic aspects, vaccine availability, and remedy research. Although the potential results, the victorious use of DL techniques to progression infected clinical images still needs a lot of time and effort and also a secure collaboration between different official higher authorities, industries, and academic groups. Also, many of the confrontations and issues linked with existing techniques as data confidentiality, the variance of outburst patterns, directives and intelligibility, and the differentiation between COVID-19 infection and non-COVID-19 indications have been discussed. At last, the study discussed the various potential indicators of DL applications in medical imaging procedures along with several other technologies as data science, cellular communications, and bio-medicine. This research work is being a great quality resource of reference and directs several novel works on medical imaging with DL in the battle aligned with COVID-19.

\section{Declarations}

Conflict of interest The authors declare no conflict of interest.

\section{References}

1. WORLDOMETER (2020) COVID-19 Coronavirus Pandemic (2020). In: WHO. https://www.worldometers.info/coronavirus/. Accessed 29 Jan 2021

2. Situation Report WHO (2020) WHO situation report-91 HIGHLIGHTS

3. Zhang Q, Yang LT, Chen Z, Li P (2018) A survey on deep learning for big data. Inf Fusion 42:146-157

4. Alam Khan F, Asif M, Ahmad A et al (2020) Blockchain technology, improvement suggestions, security challenges on smart grid 
and its application in healthcare for sustainable development. Sustain Cities Soc. https://doi.org/10.1016/j.scs.2020.102018

5. Hakak S, Khan WZ, Imran M et al (2020) Have you been a victim of COVID-19-related cyber incidents? survey, taxonomy, and mitigation strategies. IEEE Access 8:124134-124144. https:// doi.org/10.1109/ACCESS.2020.3006172

6. Kermany DS, Goldbaum M, Cai W et al (2018) Identifying medical diagnoses and treatable diseases by image-based deep learning. Cell 172:1122-1131.e9. https://doi.org/10.1016/j.cell.2018. 02.010

7. Rajaraman S, Candemir S, Kim I et al (2018) Visualization and interpretation of convolutional neural network predictions in detecting pneumonia in pediatric chest radiographs. Appl Sci. https://doi.org/10.3390/app8101715

8. Ozturk T, Talo M, Yildirim EA et al (2020) Automated detection of COVID-19 cases using deep neural networks with X-ray images. Comput Biol Med. https://doi.org/10.1016/j.compb iomed.2020.103792

9. Ahuja S, Panigrahi BK, Dey N et al (2020) Deep transfer learning-based automated detection of COVID-19 from lung CT scan slices. Appl Intell 51:571-585. https://doi.org/10.1007/ s10489-020-01826-w

10. Apostolopoulos ID, Mpesiana TA (2020) Covid-19: automatic detection from X-ray images utilizing transfer learning with convolutional neural networks. Phys Eng Sci Med 43:635-640. https://doi.org/10.1007/s13246-020-00865-4

11. Fan DP, Zhou T, Ji GP et al (2020) Inf-Net: automatic COVID-19 lung infection segmentation from CT images. IEEE Trans Med Imaging 39:2626-2637. https://doi.org/10.1109/TMI.2020.29966 45

12. Ezzat D, Hassanien AE, Ella HA (2020) An optimized deep learning architecture for the diagnosis of COVID-19 disease based on gravitational search optimization. Appl Soft Comput. https://doi.org/10.1016/j.asoc.2020.106742

13. Wang S, Kang B, Ma J, et al (2020) A deep learning algorithm using CT images to screen for corona virus disease (COVID19). medRxiv 2020.02.14.20023028. doi:https://doi.org/10.1101/ 2020.02.14.20023028

14. Shan F, Gao Y, Wang J, et al (2020) Lung infection quantification of COVID-19 in CT images with deep learning author list

15. El-Din Hemdan E, Shouman MA, Karar ME (2020) COVIDX-Net: a framework of deep learning classifiers to diagnose COVID-19 in X-ray images

16. Brinati D, Campagner A, Ferrari D, et al (2020) Detection of COVID-19 infection from routine blood exams with machine learning: a feasibility study. medRxiv

17. Hu S, Gao Y, Niu Z et al (2020) Weakly supervised deep learning for COVID-19 infection detection and classification from CT images. IEEE Access 8:118869-118883. https://doi.org/10.1109/ ACCESS.2020.3005510

18. Khan AI, Shah JL, Bhat MM (2020) CoroNet: a deep neural network for detection and diagnosis of COVID-19 from chest X-ray images. Comput Methods Programs Biomed. https://doi. org/10.1016/j.cmpb.2020.105581

19. Pereira RM, Bertolini D, Teixeira LO et al (2020) COVID-19 identification in chest X-ray images on flat and hierarchical classification scenarios. Comput Methods Programs Biomed. https:// doi.org/10.1016/j.cmpb.2020.105532

20. Narin A, Kaya C, Pamuk Z (2020) Automatic detection of coronavirus disease (COVID-19) using X-ray images and deep convolutional neural networks

21. Panwar H, Gupta PK, Siddiqui MK et al (2020) A deep learning and grad-CAM based color visualization approach for fast detection of COVID-19 cases using chest X-ray and CT-Scan images. Chaos Solitons Fractals 140:110190. https://doi.org/10.1016/j. chaos.2020.110190
22. Ni Q, Sun ZY, Qi L et al (2020) A deep learning approach to characterize 2019 coronavirus disease (COVID-19) pneumonia in chest CT images. Eur Radiol 30:6517-6527. https://doi.org/ 10.1007/s00330-020-07044-9

23. Arora V, Ng EYK, Leekha RS et al (2021) Transfer learningbased approach for detecting COVID-19 ailment in lung CT scan. Comput Biol Med 135:104575. https://doi.org/10.1016/j.compb iomed.2021.104575

24. Aslan MF, Unlersen MF, Sabanci K, Durdu A (2021) CNNbased transfer learning-BiLSTM network: a novel approach for COVID-19 infection detection. Appl Soft Comput 98:106912. https://doi.org/10.1016/j.asoc.2020.106912

25. Jangam E, Barreto AAD, Annavarapu CSR (2021) Automatic detection of COVID-19 from chest CT scan and chest X-rays images using deep learning, transfer learning and stacking. Appl Intell. https://doi.org/10.1007/s10489-021-02393-4

26. Kumar N, Gupta M, Gupta D, Tiwari S (2021) Novel deep transfer learning model for COVID-19 patient detection using X-ray chest images. J Ambient Intell Humaniz Comput. https://doi.org/ 10.1007/s12652-021-03306-6

27. Liu J, Dong B, Wang S et al (2021) COVID-19 lung infection segmentation with a novel two-stage cross-domain transfer learning framework. Med Image Anal 74:102205. https://doi.org/10. 1016/j.media.2021.102205

28. Oluwasanmi A, Aftab MU, Qin Z et al (2021) Transfer learning and semisupervised adversarial detection and classification of COVID-19 in CT images. Complexity. https://doi.org/10.1155/ 2021/6680455

29. Perumal V, Narayanan V, Rajasekar SJS (2021) Detection of COVID-19 using CXR and CT images using transfer learning and haralick features. Appl Intell 51:341-358. https://doi.org/ 10.1007/s10489-020-01831-z

30. Prakash NB, Murugappan M, Hemalakshmi GR et al (2021) Deep transfer learning for COVID-19 detection and infection localization with superpixel based segmentation. Sustain Cities Soc 75:103252. https://doi.org/10.1016/j.scs.2021.103252

31. Pham QV, Nguyen DC, Huynh-The T et al (2020) Artificial intelligence (AI) and big data for coronavirus (COVID-19) pandemic: a survey on the state-of-the-arts. IEEE Access 8:130820-130839. https://doi.org/10.1109/ACCESS.2020.3009328

32. Andersen KG, Rambaut A, Lipkin WI et al (2020) The proximal origin of SARS-CoV-2. Nat Med 26:450-452

33. WHO (2020) WHO Coronavirus Disease (COVID-19) Pandemic (2020). In: WHO. https://www.who.int/emergencies/diseases/ novel-coronavirus-2019. Accessed 30 Jan 2021

34. Shereen MA, Khan S, Kazmi A et al (2020) COVID-19 infection: origin, transmission, and characteristics of human coronaviruses. J Adv Res 24:91-98. https://doi.org/10.1016/j.jare.2020.03.005

35. Kampf G, Todt D, Pfaender S, Steinmann E (2020) Persistence of coronaviruses on inanimate surfaces and their inactivation with biocidal agents. J Hosp Infect 104:246-251. https://doi.org/10. 1016/j.jhin.2020.01.022

36. WH Organization (2020) Infection prevention and control during health care when COVID-19 is suspected. WHO

37. UNCTAD (2020) Coronavirus (COVID-19): News, analysis and resources (2020). In: WHO. https://unctad.org/programme/covid19-response. Accessed 15 Jan 2021

38. Gadekallu TR, Khare N, Bhattacharya S et al (2020) Early detection of diabetic retinopathy using pca-firefly based deep learning model. Electron. https://doi.org/10.3390/electronics9020274

39. Rodríguez A, Mendoza D, Ascuntar J, Jaimes F (2020) Supervised classification techniques for prediction of mortality in adult patients with sepsis. Am J Emerg Med. https://doi.org/10.1016/j. ajem.2020.09.013

40. Ahn E, Dagan D, Feng F, et al (2019) Unsupervised deep transfer feature learning for medical image classification 
unsupervised deep feature learning in medical images view project unsupervised deep transfer feature learning for medical image classification

41. Ge C, Gu IYH, Jakola AS, Yang J (2020) Deep semi-supervised learning for brain tumor classification. BMC Med Imaging. https://doi.org/10.1186/s12880-020-00485-0

42. Xu F, Pun CM, Li H et al (2020) Training feed-forward artificial neural networks with a modified artificial bee colony algorithm. Neurocomputing 416:69-84. https://doi.org/10.1016/j.neucom. 2019.04.086

43. Patil RS, Biradar N (2020) Automated mammogram breast cancer detection using the optimized combination of convolutional and recurrent neural network. Evol Intell. https://doi.org/10. 1007/s12065-020-00403-x

44. Greenspan H, Van Ginneken B, Summers RM (2016) Guest editorial deep learning in medical imaging: overview and future promise of an exciting new technique. IEEE Trans Med Imaging 35:1153-1159. https://doi.org/10.1109/TMI.2016.2553401

45. Alom MZ, Yakopcic C, Nasrin MS et al (2019) Breast cancer classification from histopathological images with inception recurrent residual convolutional neural network. J Digit Imaging 32:605-617. https://doi.org/10.1007/s10278-019-00182-7

46. Andreini $\mathrm{P}$, Bonechi $\mathrm{S}$, Bianchini $\mathrm{M}$ et al (2020) Image generation by GAN and style transfer for agar plate image segmentation. Comput Methods Programs Biomed. https://doi.org/10. 1016/j.cmpb.2019.105268

47. Bhattacharya S, Somayaji SRK, Gadekallu TR et al (2020) A review on deep learning for future smart cities. Internet Technol Lett. https://doi.org/10.1002/itl2.187

48. Shorfuzzaman M, Hossain MS, Alhamid MF (2021) Towards the sustainable development of smart cities through mass video surveillance: a response to the COVID-19 pandemic. Sustain Cities Soc. https://doi.org/10.1016/j.scs.2020.102582

49. Liu Y, Liu S, Wang Z (2015) A general framework for image fusion based on multi-scale transform and sparse representation. Inf Fusion 24:147-164. https://doi.org/10.1016/j.inffus.2014.09. 004

50. Ghesu FC, Krubasik E, Georgescu B et al (2016) Marginal space deep learning: efficient architecture for volumetric image parsing. IEEE Trans Med Imaging 35:1217-1228. https://doi.org/10. 1109/TMI.2016.2538802

51. Litjens G, Kooi T, Bejnordi BE et al (2017) A survey on deep learning in medical image analysis. Med Image Anal 42:60-88

52. Ker J, Wang L, Rao J, Lim T (2017) Deep learning applications in medical image analysis. IEEE Access 6:9375-9379. https:// doi.org/10.1109/ACCESS.2017.2788044

53. Zhang YD, Satapathy SC, Liu S, Li GR (2021) A five-layer deep convolutional neural network with stochastic pooling for chest CT-based COVID-19 diagnosis. In: machine vision and applications. Springer, Berlin

54. Haskins G, Kruger U, Yan P (2020) Deep learning in medical image registration: a survey. Mach Vis Appl. https://doi.org/10. 1007/s00138-020-01060-x

55. de Vos BD, Berendsen FF, Viergever MA et al (2019) A deep learning framework for unsupervised affine and deformable image registration. Med Image Anal 52:128-143. https://doi. org/10.1016/j.media.2018.11.010

56. Elmahdy MS, Jagt T, Zinkstok RT et al (2019) Robust contour propagation using deep learning and image registration for online adaptive proton therapy of prostate cancer. Med Phys 46:3329 3343. https://doi.org/10.1002/mp. 13620

57. Chee E, Wu Z (2018) AIRNet: self-supervised affine registration for 3D medical images using neural networks

58. Oktay O, Ferrante E, Kamnitsas K et al (2018) Anatomically constrained neural networks (ACNNs): application to cardiac image enhancement and segmentation. IEEE Trans Med Imaging 37:384-395. https://doi.org/10.1109/TMI.2017.2743464

59. Gu Z, Cheng J, Fu H et al (2019) CE-Net: context encoder network for 2D medical image segmentation. IEEE Trans Med Imaging 38:2281-2292. https://doi.org/10.1109/TMI.2019.29035 62

60. Guo Z, Li X, Huang H et al (2019) Deep learning-based image segmentation on multimodal medical imaging. IEEE Trans Radiat Plasma Med Sci 3:162-169. https://doi.org/10.1109/ trpms.2018.2890359

61. Seeböck P, Orlando I, Schlegl T et al (2019) Exploiting epistemic uncertainty of anatomy segmentation for anomaly detection in retinal OCT. IEEE Trans Med Imaging 39(1):87-98

62. Rajpurkar P, Irvin J, Zhu K, et al (2017) CheXNet: radiologistlevel pneumonia detection on chest X-rays with deep learning

63. Mikołajczyk A, Michał G (2018) Data augmentation for improving deep learning in image classification problem. In: IEEE

64. Shankar K, Zhang Y, Liu Y et al (2020) Hyperparameter tuning deep learning for diabetic retinopathy fundus image classification. IEEE Access 8:118164-118173. https://doi.org/10.1109/ ACCESS.2020.3005152

65. Raj RJS, Shobana SJ, Pustokhina IV et al (2020) Optimal feature selection-based medical image classification using deep learning model in internet of medical things. IEEE Access 8:5800658017. https://doi.org/10.1109/ACCESS.2020.2981337

66. Guo Y, Gao Y, Shen D (2016) Deformable MR prostate segmentation via deep feature learning and sparse patch matching. IEEE Trans Med Imaging 35:1077-1089. https://doi.org/10.1109/TMI. 2015.2508280

67. Baumgartner CF, Kamnitsas K, Matthew J et al (2016) Real-time standard scan plane detection and localisation in fetal ultrasound using fully convolutional neural networks. In: Ourselin S, Joskowicz L, Sabuncu MR et al (eds) Medical image computing and computer-assisted intervention-MICCAI 2016. Springer, New York

68. Shin SY, Lee S, Yun ID et al (2019) Joint weakly and semisupervised deep learning for localization and classification of masses in breast ultrasound images. IEEE Trans Med Imaging 38:762-774. https://doi.org/10.1109/TMI.2018.2872031

69. Xu J, Xiang L, Liu Q et al (2016) Stacked sparse autoencoder (SSAE) for nuclei detection on breast cancer histopathology images. IEEE Trans Med Imaging 35:119-130. https://doi.org/ 10.1109/TMI.2015.2458702

70. Albarqouni S, Baur C, Achilles F et al (2016) AggNet: deep learning from crowds for mitosis detection in breast cancer histology images. IEEE Trans Med Imaging 35:1313-1321. https:// doi.org/10.1109/TMI.2016.2528120

71. Shin HC, Roth HR, Gao M et al (2016) Deep convolutional neural networks for computer-aided detection: $\mathrm{CNN}$ architectures, dataset characteristics and transfer learning. IEEE Trans Med Imaging 35:1285-1298. https://doi.org/10.1109/TMI.2016.25281 62

72. Liao F, Liang M, Li Z et al (2019) Evaluate the malignancy of pulmonary nodules using the 3-D deep leaky noisy-OR network. IEEE Trans Neural Netw Learn Syst 30:3484-3495. https://doi. org/10.1109/TNNLS.2019.2892409

73. Duan J, Bello G, Schlemper J et al (2019) Automatic 3D biventricular segmentation of cardiac images by a shape-refined multi- task deep learning approach. IEEE Trans Med Imaging 38:2151-2164. https://doi.org/10.1109/TMI.2019.2894322

74. Shin HC, Orton MR, Collins DJ et al (2013) Stacked autoencoders for unsupervised feature learning and multiple organ detection in a pilot study using 4D patient data. IEEE Trans Pattern Anal Mach Intell 35:1930-1943. https://doi.org/10.1109/TPAMI. 2012.277 
75. Bai W, Shi W, O'Regan DP et al (2013) A probabilistic patchbased label fusion model for multi-atlas segmentation with registration refinement: application to cardiac MR images. IEEE Trans Med Imaging 32:1302-1315. https://doi.org/10.1109/TMI. 2013.2256922

76. Cruz-Roa AA, Ovalle JEA, Madabhushi A, Osorio FAG (2013) LNCS 8150 - a deep learning architecture for image representation, visual interpretability and automated basal-cell carcinoma cancer detection

77. Shen W, Zhou M, Yang C, Tian J (2015) Multi-scale convolutional neural networks for lung nodule classification. In: Ourselin S, Alexander DC, Westin C-F, Cardoso MJ (eds) Information processing in medical imaging. Springer, New York

78. Payer C, Stern D, Bischof H, Urschle M (2016) regressing heatmaps for multiple landmark localization using CNNs. In: Ourselin S, Joskowicz L, Sabuncu MR et al (eds) Medical image computing and computer-assisted intervention - MICCAI 2016. Springer, New York

79. Pratt H, Coenen F, Broadbent DM et al (2016) Convolutional neural networks for diabetic retinopathy. Procedia computer science. Elsevier, Amsterdam, pp 200-205

80. Abràmoff MD, Lou Y, Erginay A et al (2016) Improved automated detection of diabetic retinopathy on a publicly available dataset through integration of deep learning. Investig Ophthalmol Vis Sci 57:5200-5206. https://doi.org/10.1167/iovs.16-19964

81. Kawahara J, Hamarneh G (2016) Multi-resolution-tract CNN with hybrid pretrained and skin-lesion trained layers. In: Wang L, Adeli E, Wang Q et al (eds) Machine learning in medical imaging. Springer, New York

82. Zhu Q, Du B, Yan P (2020) Boundary-weighted domain adaptive neural network for prostate MR image segmentation. IEEE, pp. 753-763

83. Qiao L, Zhu Y, Zhou H (2020) Diabetic retinopathy detection using prognosis of microaneurysm and early diagnosis system for non-proliferative diabetic retinopathy based on deep learning algorithms. IEEE Access 8:104292-104302. https://doi.org/10. 1109/ACCESS.2020.2993937

84. Komatsu M, Sakai A, Komatsu R et al (2021) Detection of cardiac structural abnormalities in fetal ultrasound videos using deep learning. Appl Sci 11:1-12. https://doi.org/10.3390/app11 010371

85. Pan F, Li L, Liu B et al (2021) A novel deep learning-based quantification of serial chest computed tomography in Coronavirus disease 2019 (COVID-19). Sci Rep. https://doi.org/10.1038/ s41598-020-80261-w

86. Bhattacharya S, Reddy Maddikunta PK, Pham QV et al (2021) Deep learning and medical image processing for coronavirus (COVID-19) pandemic: a survey. Sustain Cities Soc. https://doi. org/10.1016/j.scs.2020.102589

87. Katsamenis I, Protopapadakis E, Voulodimos A, et al (2020) Transfer learning for COVID-19 pneumonia detection and classification in chest X-ray images. medRxiv 2020.12.14.20248158. doi:https://doi.org/10.1101/2020.12.14.20248158

88. Fang Y, Zhang H, Xie J et al (2020) Sensitivity of chest CT for COVID-19: comparison to RT-PCR. Radiology 296:E115-E117

89. Wu Z, McGoogan JM (2020) Characteristics of and important lessons from the coronavirus disease 2019 (COVID-19) outbreak in China: summary of a report of 72314 cases from the chinese center for disease control and prevention. JAMA - J Am Med Assoc 323:1239-1242

90. Velavan TP, Meyer CG (2020) The COVID-19 epidemic. Trop Med Int Heal 25:278-280

91. Suzuki K (2017) Overview of deep learning in medical imaging. Radiol Phys Technol 10:257-273
92. Lundervold AS, Lundervold A (2019) An overview of deep learning in medical imaging focusing on MRI. Z Med Phys 29:102-127

93. Anwar SM, Majid M, Qayyum A et al (2018) Medical image analysis using convolutional neural networks: a review. J Med Syst 42:1-13

94. Cao C, Liu F, Tan H et al (2018) Deep learning and its applications in biomedicine. Genomics Proteomics Bioinforma 16:17-32

95. Ardabili SF, Mosavi A, Ghamisi P, et al (2020) COVID-19 outbreak prediction with machine learning. medRxiv

96. OurWorldInData, Max R, Hannah R, Esteban OO, Joe H (2020) Coronavirus pandemic (COVID-19). In: OurWorldInData.org. https://ourworldindata.org/coronavirus. Accessed 29 Jan 2021

97. WHO (2020) WHO Coronavirus disease (COVID-19) dashboard (2020). In: WHO. https://covid19.who.int/. Accessed 29 Jan 2021

98. Ting DSW, Carin L, Dzau V, Wong TY (2020) Digital technology and COVID-19. Nat Med 26:459-461

99. Tajbakhsh N, Jeyaseelan L, Li Q et al (2020) Embracing imperfect datasets: a review of deep learning solutions for medical image segmentation. Med Image Anal. https://doi.org/10.1016/j. media.2020.101693

100. Wu D, Wu T, Liu Q, Yang Z (2020) The SARS-CoV-2 outbreak: what we know. Int J Infect Dis 94:44-48

101. Huang C, Wang Y, Li X et al (2020) Clinical features of patients infected with 2019 novel coronavirus in Wuhan, China. Lancet 395:497-506. https://doi.org/10.1016/S0140-6736(20)30183-5

102. Husnayain A, Fuad A, Su ECY (2020) Applications of google search trends for risk communication in infectious disease management: a case study of the COVID-19 outbreak in Taiwan. Int J Infect Dis 95:221-223. https://doi.org/10.1016/j.ijid.2020.03. 021

103. Remuzzi A, Remuzzi G (2020) COVID-19 and Italy: what next? Lancet 395:1225-1228

104. Liu D, Clemente L, Poirier C et al (2020) Correction: real-time forecasting of the COVID-19 outbreak in chinese provinces: machine learning approach using novel digital data and estimates from mechanistic models. J Med Internet Res 22:e23996

105. Zhou Y, Yang Z, Guo Y, et al (2020) A new predictor of disease severity in patients with COVID-19 in Wuhan, China. medRxiv

106. Bernheim A, Mei X, Huang M et al (2020) Chest CT findings in coronavirus disease 2019 (COVID-19): relationship to duration of infection. Radiology 295:685-691

107. Yildirim O, Talo M, Ay B et al (2019) Automated detection of diabetic subject using pre-trained 2D-CNN models with frequency spectrum images extracted from heart rate signals. Comput Biol Med 113:103387. https://doi.org/10.1016/j.compb iomed.2019.103387

108. Saba T, Sameh Mohamed A, El-Affendi M et al (2020) Brain tumor detection using fusion of hand crafted and deep learning features. Cogn Syst Res 59:221-230. https://doi.org/10.1016/j. cogsys.2019.09.007

109. Dorj UO, Lee KK, Choi JY, Lee M (2018) The skin cancer classification using deep convolutional neural network. Multimed Tools Appl 77:9909-9924. https://doi.org/10.1007/ s11042-018-5714-1

110. Ribli D, Horváth A, Unger Z et al (2018) Detecting and classifying lesions in mammograms with deep learning. Sci Rep 8:1-7. https://doi.org/10.1038/s41598-018-22437-z

111. Celik Y, Talo M, Yildirim O et al (2020) Automated invasive ductal carcinoma detection based using deep transfer learning with whole-slide images. Pattern Recognit Lett 133:232-239. https://doi.org/10.1016/j.patrec.2020.03.011

112. Jmour N, Zayen S, Abdelkrim A (2018) Convolutional neural networks for image classification. In: 2018 international conference 
on advanced systems and electric technologies (IC_ASET). pp 397-402. doi:https://doi.org/10.1109/ASET.2018.8379889

113. Lecun Y, Bengio Y, Hinton G (2015) Deep learning. Nature 521:436-444. https://doi.org/10.1038/nature14539

114. Sriastava N, Hinton G, Krizhevsky A et al (2016) Dropout: a simple way to prevent neural networks from overfitting. Int Conf Adv Electr Electron Syst Eng ICAEES 15:520-525

115. Wu Z, Shen C, van den Hengel A (2019) Wider or deeper: Revisiting the resnet model for visual recognition. Pattern Recognit 90:119-133. https://doi.org/10.1016/j.patcog.2019.01.006

116. Russakovsky O, Deng J, Su H et al (2015) ImageNet large scale visual recognition challenge. Int J Comput Vis 115:211-252. https://doi.org/10.1007/s11263-015-0816-y

117. Ahn JM, Kim S, Ahn KS et al (2018) A deep learning model for the detection of both advanced and early glaucoma using fundus photography. PLoS ONE 13:1-8. https://doi.org/10.1371/journ al.pone.0207982

118. Byra M, Styczynski G, Szmigielski C et al (2018) Transfer learning with deep convolutional neural network for liver steatosis assessment in ultrasound images. Int J Comput Assist Radiol Surg 13:1895-1903. https://doi.org/10.1007/s11548-018-1843-2

119. SIRM Database. https://sirm.org/category/senza-categoria/covid19/\%0A\%0A. Accessed 21 Aug 2021

120. Radiopaedia database. https://radiopaedia.org/articles/covid19-4?lang=us\%0A\%0A. Accessed 21 Aug 2021

121. MosMed. https://mosmed.ai/datasets/covid19_1110/\%0A\%0A. Accessed 21 Aug 2021

122. UCSD database. https://github.com/UCSD-AI4H/COVID-CT. Accessed 21 Aug 2021

123. COVID-19 ML Database. https://github.com/ieee8023/covidchestxray-dataset. Accessed 21 Aug 2021

124. COVID-19 CT segmentation database 1. http://medicalsegmenta tion.com/covid19/. Accessed 21 Aug 2021

125. COVID-19 CT segmentation database 2. http://medicalsegmenta tion.com/covid19/. Accessed 21 Aug 2021

126. COVID-19 CT slices and infection segmentation. https://zenodo. org/record/3757476\#.YSDS6Y4zbIV. Accessed 21 Aug 2021

127. AIforCOVID. https://aiforcovid.radiomica.it/. Accessed 21 Aug 2021

128. Eurorad. https://www.eurorad.org/advanced-search? search= COVID. Accessed 21 Aug 2021

129. Wu JT, Leung K, Leung GM (2020) Nowcasting and forecasting the potential domestic and international spread of the 2019-nCoV outbreak originating in Wuhan, China: a modelling study. Lancet 395:689-697. https://doi.org/10.1016/S0140-6736(20)30260-9

130. Rothan HA, Byrareddy SN (2020) The epidemiology and pathogenesis of coronavirus disease (COVID-19) outbreak. J Autoimmun 109:102433

131. Surveillances (2020) The epidemiological characteristics of an outbreak of 2019 novel coronavirus diseases (COVID-19) in China. Epidemiol Work Gr NCIP Epidemic Response, Chinese Cent Dis Control Prev 41:145-151

132. Lu R, Zhao X, Li J et al (2020) Genomic characterisation and epidemiology of 2019 novel coronavirus: implications for virus origins and receptor binding. Lancet 395:565-574. https://doi. org/10.1016/S0140-6736(20)30251-8

133. Xu X, Chen P, Wang J et al (2020) Evolution of the novel coronavirus from the ongoing Wuhan outbreak and modeling of its spike protein for risk of human transmission. Sci China Life Sci 63:457-460

134. Bassetti M, Vena A, Giacobbe DR (2020) The novel chinese coronavirus (2019-nCoV) infections: challenges for fighting the storm. Eur J Clin Invest 50

135. Malik YS, Kumar P, Sircar S, et al (2020) Pandemic coronavirus disease (COVID-19): challenges and a global perspective. doi:https://doi.org/10.20944/preprints202004.0469.v1
136. Vision (2020) Coronavirus outbreak can machine vision and imaging play a part? (2020). In: Vision Syst. Des. https://www. vision-systems.com/home/article/14170078/coronavirus-outbr eak-can-machine-vision-and-imaging-play-a-part

137. Ji W, Wang W, Zhao X, et al (2020) Homologous recombination within the spike glycoprotein of the newly identified coronavirus may boost cross-species transmission from snake to human

138. Kvalsvig A, Barnard LT, Gray L, et al (2020) Supporting the COVID-19 pandemic response: surveillance and outbreak analytics prepared for the ministry of health

139. Pourghasemi HR, Pouyan S, Farajzadeh Z, et al (2020) Assessment of the outbreak risk, mapping and infestation behavior of COVID-19: Application of the autoregressive and moving average (ARMA) and polynomial models. medRxiv

140. Saha A, Gupta K, Patil M (2020) Monitoring and epidemiological trends of coronavirus disease (COVID-19) around the world

141. CSSE C for SSJHUE (2020) Center for systems science engineering (CSSE) COVID-19 dashboard (2020). In: Johns H, Univ. Med. https://coronavirus.jhu.edu/map.html. Accessed 30 Jan 2021

142. Boulos MNK, Geraghty EM (2020) Geographical tracking and mapping of coronavirus disease COVID-19/severe acute respiratory syndrome coronavirus 2 (SARS-CoV-2) epidemic and associated events around the world: How 21st century GIS technologies are supporting the global fight against outbr. Int J Health Geogr. https://doi.org/10.1186/s12942-020-00202-8

143. G.O.I NIC MEITY Aarogya setu (2020) Government of India NIC, MEITY Aarogya Setu App (2020). In: mygov.in. https:// www.mygov.in/aarogya-setu-app/. Accessed 30 Jan 2021

144. Chen J, Wu L, Zhang J, et al (2020) Deep learning-based model for detecting 2019 novel coronavirus pneumonia on high-resolution computed tomography: a prospective study. medRxiv

145. Wang L, Wang Y, Ye D, Liu Q (2020) Review of the 2019 novel coronavirus (SARS-CoV-2) based on current evidence. Int J Antimicrob Agents. https://doi.org/10.1016/j.ijantimicag.2020. 105948

146. Rahman MA, Zaman N, Asyhari AT et al (2020) Data-driven dynamic clustering framework for mitigating the adverse economic impact of Covid-19 lockdown practices. Sustain Cities Soc. https://doi.org/10.1016/j.scs.2020.102372

147. Hopman J, Allegranzi B, Mehtar S (2020) Managing COVID-19 in low- and middle-income countries. JAMA - J Am Med Assoc 323:1549-1550

148. Das S, Ghosh P, Sen B, Mukhopadhyay I (2020) Critical community size for COVID-19 - a model based approach to provide a rationale behind the lockdown

149. Singh R, Adhikari R (2020) Age-structured impact of social distancing on the COVID-19 epidemic in India

150. Ai T, Yang Z, Hou H et al (2020) Correlation of chest CT and RT-PCR testing for coronavirus disease 2019 (COVID-19) in China: a report of 1014 cases. Radiology 296:E32-E40. https:// doi.org/10.1148/radiol.2020200642

151. Bai HX, Hsieh B, Xiong $Z$ et al (2020) Performance of radiologists in differentiating COVID-19 from non-COVID-19 viral pneumonia at chest CT. Radiology 296:E46-E54. https://doi.org/ 10.1148/radiol.2020200823

152. Chen N, Zhou M, Dong X et al (2020) Epidemiological and clinical characteristics of 99 cases of 2019 novel coronavirus pneumonia in Wuhan, China: a descriptive study. Lancet 395:507513. https://doi.org/10.1016/S0140-6736(20)30211-7

153. Peng QY, Wang XT, Zhang LN (2020) Findings of lung ultrasonography of novel corona virus pneumonia during the 20192020 epidemic. Intensive Care Med 46:849-850. https://doi.org/ 10.1007/s00134-020-05996-6

154. Poggiali E, Dacrema A, Bastoni D et al (2020) Can lung US help critical care cliniciansin the early diagnosis of novel coronavirus 
(COVID-19) pneumonia? Radiology 295:715-721. https://doi. org/10.1148/radiol.2020200370

155. Qin C, Liu F, Yen TC, Lan X (2020) 18F-FDG PET/CT findings of COVID-19: a series of four highly suspected cases. Eur J Nucl Med Mol Imaging 47:1281-1286. https://doi.org/10.1007/ s00259-020-04734-w

156. Zou S, Zhu X (2020) FDG PET/CT of COVID-19. Radiology 296:E118

157. Jin YH, Cai L, Cheng ZS et al (2020) A rapid advice guideline for the diagnosis and treatment of 2019 novel coronavirus (2019-nCoV) infected pneumonia (standard version). Mil Med Res 7:1-23

158. Xu X, Jiang X, Ma C et al (2020) A deep learning system to screen novel coronavirus disease 2019 pneumonia. Engineering 6:1122-1129. https://doi.org/10.1016/j.eng.2020.04.010

159. WHO (2020) WHO Public statement for collaboration on COVID-19 vaccine development. In: WHO. https://www.who. int/news/item/13-04-2020-public-statement-for-collaborationon-covid-19-vaccine-development. Accessed 15 Jan 2021

160. Kannan S, Subbaram K, Ali S, Kannan H (2020) The role of artificial intelligence and machine learning techniques: race for COVID-19 vaccine. Arch Clin Infect Dis. https://doi.org/10. 5812/archcid.103232

161. Qiao R, Tran NH, Shan B, et al (2020) Personalized workflow to identify optimal T-cell epitopes for peptide-based vaccines against COVID-19

162. Tayebi $Z$ (2020) Machine learning and deep learning to predict cross-machine learning and deep learning to predict cross-immunoreactivity of viral epitopes immunoreactivity of viral epitopes

163. Vaishya R, Javaid M, Khan IH, Haleem A (2020) Artificial intelligence (AI) applications for COVID-19 pandemic. Diabetes Metab Syndr Clin Res Rev 14:337-339. https://doi.org/10. 1016/j.dsx.2020.04.012

164. Zhang H, Saravanan KM, Yang Y et al (2020) Deep learning based drug screening for novel coronavirus 2019-nCov. Interdiscip Sci Comput Life Sci 12:368-376. https://doi.org/10.1007/ s12539-020-00376-6

165. Beck BR, Shin B, Choi Y et al (2020) Predicting commercially available antiviral drugs that may act on the novel coronavirus (SARS-CoV-2) through a drug-target interaction deep learning model. Comput Struct Biotechnol J 18:784-790. https://doi.org/ 10.1016/j.csbj.2020.03.025

166. Ong E, Wong MU, Huffman A, He Y (2020) COVID-19 coronavirus vaccine design using reverse vaccinology and machine learning. bioRxiv
167. WHO (2020) WHO COVID-19 vaccine. In: WHO. https://www. who.int/emergencies/diseases/novel-coronavirus-2019/covid-19vaccines. Accessed 20 Jan 2021

168. Vaid S, Kalantar R, Bhandari M (2020) Deep learning COVID19 detection bias: accuracy through artificial intelligence. Int Orthop Springer. https://doi.org/10.1007/s00264-020-04609-7

169. Heidari M, Mirniaharikandehei S, Khuzani AZ et al (2020) Improving the performance of $\mathrm{CNN}$ to predict the likelihood of COVID-19 using chest X-ray images with preprocessing algorithms. Int J Med Inform. https://doi.org/10.1016/j.ijmedinf. 2020.104284

170. Sun L, Mo Z, Yan F et al (2020) Adaptive feature selection guided deep forest for COVID-19 classification with chest CT. IEEE J Biomed Health Inform 24:2798-2805. https://doi.org/10. 1109/JBHI.2020.3019505

171. Punn NS, Agarwal S (2020) Automated diagnosis of COVID19 with limited posteroanterior chest X-ray images using finetuned deep neural networks. Appl Intell. https://doi.org/10.1007/ s10489-020-01900-3

172. Wang L, Lin ZQ, Wong A (2020) COVID-Net: a tailored deep convolutional neural network design for detection of COVID-19 cases from chest X-ray images. Sci Rep 10:1-12. https://doi.org/ $10.1038 / \mathrm{s} 41598-020-76550-Z$

173. Song Y, Zheng S, Li L et al (2021) Deep learning enables accurate diagnosis of novel coronavirus (COVID-19) with CT images. IEEE/ACM Trans Comput Biol Bioinform. https://doi.org/10. 1109/TCBB.2021.3065361

174. Rahimzadeh M, Attar A (2020) A modified deep convolutional neural network for detecting COVID-19 and pneumonia from chest X-ray images based on the concatenation of Xception and ResNet50V2. Inform Med Unlocked 19:100360. https://doi.org/ 10.1016/j.imu.2020.100360

175. Bos ECW, Heijnen L, Luytjes W, Spaan WJM (1995) Mutational analysis of the murine coronavirus spike protein: effect on cellto-cell fusion. Virology 214(2):453-463

176. Zhuang F, Qi Z, Duan K, et al (2019) A Comprehensive Survey on Transfer Learning

177. Rehman A, Naz S, Khan A, et al (2020) Improving coronavirus (COVID-19) diagnosis using deep transfer learning. medRxiv

Publisher's Note Springer Nature remains neutral with regard to jurisdictional claims in published maps and institutional affiliations. 